\title{
Quenching timescales of galaxies in the EAGLE simulations
}

\author{
Ruby J. Wright ${ }^{1,2}$, Claudia del P. Lagos ${ }^{1,2}$, Luke J. M. Davies ${ }^{1}$, Chris Power $^{1,2}$, \\ James W. Trayford ${ }^{3}$, O. Ivy Wong ${ }^{1,2}$ \\ ${ }^{1}$ International Centre for Radio Astronomy Research (ICRAR), M468, University of Western Australia, 35 Stirling Hwy, Crawley, \\ WA 6009, Australia \\ ${ }^{2}$ ARC Centre of Excellence for All Sky Astrophysics in 3 Dimensions (ASTRO 3D). \\ ${ }^{3}$ Leiden Observatory, Leiden University, PO Box 9513, NL-2300 RA Leiden, the Netherlands.
}

Accepted XXX. Received YYY; in original form ZZZ

\begin{abstract}
We use the EAGLE simulations to study the connection between the quenching timescale, $\tau_{\mathrm{Q}}$, and the physical mechanisms that transform star-forming galaxies into passive galaxies. By quantifying $\tau_{\mathrm{Q}}$ in two complementary ways - as the time over which (i) galaxies traverse the green valley on the colour-mass diagram, or (ii) leave the main sequence of star formation and subsequently arrive on the passive cloud in specific star formation rate (SSFR)-mass space - we find that the $\tau_{\mathrm{Q}}$ distribution of high-mass centrals, low-mass centrals and satellites are divergent. In the low stellar mass regime where $M_{\star}<10^{9.6} M_{\odot}$, centrals exhibit systematically longer quenching timescales than satellites $(\approx 4$ Gyr compared to $\approx 2$ Gyr $)$. Satellites with low stellar mass relative to their halo mass cause this disparity, with ram pressure stripping quenching these galaxies rapidly. Low mass centrals are quenched as a result of stellar feedback, associated with long $\tau_{\mathrm{Q}} \gtrsim 3 \mathrm{Gyr}$. At intermediate stellar masses where $10^{9.7} \mathrm{M}_{\odot}<\mathrm{M}_{\star}<10^{10.3} \mathrm{M}_{\odot}, \tau_{\mathrm{Q}}$ are the longest for both centrals and satellites, particularly for galaxies living in groups with other gas-rich galaxies. At $M_{\star} \gtrsim 10^{10.3} \mathrm{M}_{\odot}$, galaxy merger counts and black hole activity increase steeply for all galaxies. Quenching timescales for centrals and satellites decrease with stellar mass in this regime to $\tau_{\mathrm{Q}} \lesssim 2$ Gyr. In anticipation of new intermediate redshift observational galaxy surveys, we analyse the passive and star-forming fractions of galaxies across redshift, and find that the $\tau_{\mathrm{Q}}$ peak at intermediate stellar masses is responsible for a peak (inflection point) in the fraction of green valley central (satellite) galaxies at $z \approx 0.5-0.7$.
\end{abstract}

Key words: galaxies: formation - galaxies: evolution

\section{INTRODUCTION}

Large observational galaxy surveys have demonstrated a distinct bimodality in the galaxy colour-magnitude diagram (CMD) (e.g. Strateva et al. (2001); Baldry et al. (2006); Wyder et al. (2007); Taylor et al. (2015)). At low redshift, two populations of galaxies are separated in colour, denoted the red sequence and the blue cloud. The red sequence is tight, and home to galaxies which are typically early-type and show low star formation rates. The blue cloud is comparatively more dispersed, and contains galaxies which are typically late-type and have higher star formation rates (Baldry et al. 2004; Driver et al. 2006; Schiminovich et al. 2007). Residing between these two populations is a region denoted the green valley, which appears in observations to be consistently less densely populated than the two aforementioned populations (Martin et al. 2007; Salim 2014). Work by Eales et al. (2018), however, indicates that this well-define under- density may be due to observational biases. Galaxy bimodality can also be observed in SSFR-stellar mass space (Wyder et al. 2007), where the blue cloud population in colour-mass space is correlated with the main sequence population in the SSFR-stellar mass plane (Noeske et al. 2007; Davies et al. 2016b).

Since the green valley is nominally found to be sparsely populated, it has been theorised that galaxies evolve from the blue cloud to the red sequence rapidly as their star formation rates slow, the colour transformation being indicative of a process broadly referred to as star formation quenching (Fang et al. 2013; Salim 2014; Peng et al. 2015; Bremer et al. 2018). Star formation quenching can be the result of one or a combination of mechanisms which can be classified as internal or external. An internal quenching mechanism stifles star formation due to some galaxylocalised process, while an external quenching mechanism slows star formation in a galaxy by some environmentally- 
driven effect. Internal quenching mechanisms include, but are not limited to: active galactic nuclei (AGN) activity (Di Matteo et al. 2005; Murray et al. 2005; Croton et al. 2006; Bower et al. 2006; Hopkins et al. 2006; Lagos et al. 2008), supernovae feedback (Springel et al. 2005; Cox et al. 2006b; Dalla Vecchia \& Schaye 2012; Lagos et al. 2013), and virial shock heating (Birnboim \& Dekel 2003; Kereš et al. 2009). External quenching mechanisms include, but are not limited to: major \& minor mergers (Toomre \& Toomre 1972; Springel 2005; Cox et al. 2006a; McNamara et al. 2006), ram pressure stripping (Gunn \& Gott 1972; Knobel et al. 2013; Brown et al. 2017), and galaxy strangulation (Larson et al. 1980; Balogh et al. 2000; Knobel et al. 2013; Peng et al. 2015). Although we make a clear distinction between these quenching mechanisms, the reader should note that quenching mechanisms can be, and often are, interrelated in non-linear manner (Hopkins 2008). Most notable is the relationship between AGN activity and galaxy merging events, where angular momentum dissipation acts to funnel matter towards a galaxy's central super-massive black hole (SMBH) and up-regulate black hole accretion rates (Barnes \& Hernquist 1991; Mihos \& Hernquist 1996; Hopkins et al. 2008; Kocevski et al. 2012). Regarding environmental stripping, Jung et al. (2018) recently investigated how ram pressure stripping can induce an excess of gas poor galaxies in clusters, and concluded that there are fast (characterised by radial orbits and stripping during the first pass of a galaxy through a cluster) and slow (characterised by circular orbits and slower stripping) pathways for galaxies to lose gas. Which pathway a galaxy takes is determined by its gas content at interface with the cluster. Each of the aforementioned quenching mechanisms can affect galaxies in diverse ways, and in principle, some could act over different, distinctive timescales. This makes the results of Bremer et al. (2018) of a relatively universal green-valley crossing timescale somewhat puzzling.

Earlier work using Sloan Digital Sky Survey (SDSS) data by Schawinski et al. (2014) showed that there may be two different pathways that galaxies take in quenching and traversing the green valley, characterised by different quenching timescales $\left(\tau_{\mathrm{Q}}\right)$ : the time it takes for a galaxy to transform from star-forming to passive. Early-type galaxies were found to have shorter quenching timescales $\left(\tau_{\mathrm{Q}}<250\right.$ Myr), while late-type galaxies would quench more slowly with timescales $\tau_{\mathrm{Q}} \approx 1 \mathrm{Gyr}$. These figures were derived by constraining $\frac{d}{d t}$ (SSFR) and using an exponentially declining star formation model. They claim that the key difference between the two quenching pathways, and corresponding quenching timescales, is how the star-forming gas reservoir in a galaxy changes. The assumption adopted is that any quenching mechanism acts by depleting the gas reservoir in a galaxy available for star formation, and that the quenching mechanism will determine the quenching timescale of a galaxy (Davé et al. 2012; Lilly et al. 2013). This idea commonly referred to as the "bathtub" model, explaining how the gas reservoir in a galaxy can change over time through various inflows and outflows.

Moving away from observational studies, multiple large scale projects involving the hydrodynamical simulation of galaxy formation have found great success in reproducing several observations of galaxy populations, in recent years becoming state-of-the-art in their capabilities as predictive tools for extragalactic astrophysics. Such simulations include EAGLE (Schaye et al. 2015; Crain et al. 2015; McAlpine et al. 2016), Illustris-TNG (Pillepich et al. 2018), Horizon-AGN (Dubois et al. 2016), MUFASA (Davé et al. 2016), and others.

However, there has been some tension in the results arising from these new simulations concerning the color bimodality and quenching timescales of galaxies. Trayford et al. (2016) used EAGLE to study the colour evolution of galaxies, concluding that galaxies join the red sequence because either (1) they become satellites in a larger system (the path affecting mostly lower mass galaxies), or (2) because of AGN feedback (the path affecting mostly higher mass galaxies). Using the Horizon-AGN simulation, Dubois et al. (2016) show that star formation quenching, galaxy morphology, and cosmic gas accretion are intricately related, where early-type galaxies require AGN feedback to down-regulate cosmic gas infall and cease star-formation to regrow disk structures (see also Sparre \& Springel 2017). These different mechanisms act on different stellar mass ranges, and should naturally lead to different quenching timescales.

In apparent contrast to these conclusions, Nelson et al. (2018) found in the Illustris-TNG simulations that the colour transition timescale of galaxies (using the colour-mass diagram) was unimodal, on average $\approx 1.6 \mathrm{Gyr}$ over all stellar mass bins from $M_{\star}=10^{9} \mathrm{M}_{\odot}$ to $10^{12.5} \mathrm{M}_{\odot}$, with higher mass galaxies typically showing shorter quenching timescales. The unimodal distribution of quenching timescales they observed opposes the existence of multiple colour transition pathways with distinct physical timescales. This said, their work does demonstrate a small peak in quenching timescales in for $M_{\star} \approx 10{ }^{10} M_{\odot}$ for the TNG100-1 run for both centrals and satellites. They also found that in general, the behaviour of centrals and satellites in their colour transition were very similar.

The aim of this work is to study the quenching timescales of galaxies produced by the EAGLE suite of cosmological simulations using multiple methods, and to connect the distribution of $\tau_{\mathrm{Q}}$ to the physical mechanisms which stifle star formation. In order to maximise the robustness of our approach, we employ two very different methods of quantifying $\tau_{\mathrm{Q}}$ : (i) adopting a similar methodology to that of Trayford et al. (2016); Nelson et al. (2018) to calculate $\tau_{\mathrm{Q}}$, by analysing the movement of galaxies through colour-mass space, and (ii) making use of the SSFR-stellar mass plane. The diverse galaxy population available in EAGLE makes this simulation (and numerical models of cosmological galaxy formation in general) an ideal test-bed for identifying and quantifying how certain physical mechanisms would produce changes in the distribution of quenching timescale. In doing so, we add to the developing body of knowledge looking to explain how galaxy colour bimodality in the local Universe has developed, from a mechanistic perspective.

The content we present in this paper is structured as follows. In $\S 2$ we introduce the EAGLE simulation suite and subgrid models that are relevant to this study, as well as how specific physical quantities were extracted from the database and post-processed. $\S 3$ isolates the effect of external and internal mechanisms on quenching timescale distributions. In $\S 4$ we conclude what these findings tell us about the physical mechanisms acting to quench different galaxies. 


\section{THE EAGLE SIMULATIONS}

Studying quenching timescales using a simulation based approach is ideal as it allows for tracking of individual galactic properties over time. The EAGLE (Evolution and Assembly of GaLaxies and their Environments) simulation suite (Schaye et al. 2015; Crain et al. 2015) is a collection of cosmological hydrodynamical simulations which follow the evolution of galaxies and cosmological structure from $z=20$ to $z=0$. In EAGLE, dark matter (DM) halos are identified using a field-of-friends (FOF) approach (Davis et al. 1985), and subsequently the SUBFIND algorithm (Springel et al. 2001; Dolag et al. 2009) identifies overdensities of particles within these structures, corresponding to galaxies. The ANARCHY (Schaller et al. 2015) set of refinements were implemented on the GADGET-3 tree-SPH (smoothed particle hydrodynamics) code (Springel et al. 2005) to perform the EAGLE simulations over a variety of periodic volumes, at a number of numerical resolutions. EAGLE adopts the parameters of a $\Lambda \mathrm{CDM}$ universe from Planck Collaboration et al. (2014), and the initial conditions outlined in Jenkins (2013). Twenty-nine discrete snapshots of pertinent simulation and post-processed galaxy integrated quantities were taken in each simulation run. For our study, we make use of the RefL0100N1504 EAGLE simulation run, which has a co-moving box size of $100 \mathrm{cMpc}$, the largest of the suite (Schaye et al. 2015). This run has $1504^{3}$ DM particles, and the same initial number of gas particles. For more details see Schaye et al. (2015).

Sub-grid physics modules were implemented to treat the physics which are important for galaxy formation and evolution but that happen below the resolution of the simulation. These include: (i) radiative cooling and photoheating, (ii) star formation, (iii) stellar evolution and enrichment, (iv) stellar feedback, and (v) SMBH growth and AGN feedback. In the next section we provide a brief description of how these mechanisms are modelled in EAGLE.

\subsection{Subgrid physics}

In an element-by-element approach, photo-heating and radiative cooling were applied based on the work of Wiersma et al. (2009). This included the effect of 11 elements which were deemed influential: $\mathrm{H}, \mathrm{He}, \mathrm{C}, \mathrm{N}, \mathrm{O}, \mathrm{Ne}, \mathrm{Mg}, \mathrm{Si}, \mathrm{S}, \mathrm{Ca}$, and Fe (Schaye et al. 2015). The effect of radiation from the UV and X-ray background described by Haardt \& Madau (2001) was implemented on each element individually.

Star formation occurs almost exclusively in cold interstellar medium (ISM) gas. Since the EAGLE simulations do not provide the resolution to model cold, interstellar gas on the required scale, a metallicity dependent density threshold is set, above which, star formation was locally permitted (Schaye et al. 2015). The simulation also imposes a temperature floor of $8,000 \mathrm{~K}$, recognising that the cold gas phase was not modelled effectively (Schaye et al. 2015). Gas particles are converted to star particles stochastically, with the star formation rate based on tuning a pressure law (Schaye \& Dalla Vecchia 2008) to observations of the relationship between gas and star formation rate surface densities (Kennicutt 1998) at $z=0$. Also considered was the energy feedback from star formation in the form of stellar winds, radiation, and supernovae. This involved a purely thermal energy in- jection in the form of a temperature boost, $\Delta T_{\mathrm{SF}}=10^{6} \mathrm{~K}$, based on the work of Dalla Vecchia \& Schaye (2012), which acts to heat the gas particles nearby newly formed stars.

Often a distinction is made between quasar-mode and radio-mode galaxies host to AGN (Croton et al. 2006), based on their SMBH accretion efficiency. In EAGLE, a fixed efficiency was adopted to reduce the number of feedback channels (Schaye et al. 2015). This singular efficiency is closest physically to the more active "quasar-mode" AGN, however acting as a maintainance mode in the practice (see Bower et al. (2017) for a discussion). SMBHs are seeded in EAGLE when a dark matter halo exceeds a virial mass of $10^{10} \mathrm{~h}^{-1} \mathrm{M}_{\odot}$, with the seed SMBHs having an initial mass of $10^{5} \mathrm{~h}^{-1} \mathrm{M}_{\odot}$. Subsequently, SMBHs can grow via Eddingtonlimited-accretion (Schaye et al. 2015), as well as mergers with other SMBHs, according to work by Springel (2005). AGN feedback in EAGLE involves a purely thermal energy injection into its surroundings. Similar to star formation feedback, this took the form of a purely thermal temperature boost, chosen to be $\Delta T_{\mathrm{BH}}=10^{8.5} \mathrm{~K}$ (Schaye et al. 2015).

\subsection{Measuring quenching timescales in EAGLE}

We measure the quenching timescale, $\tau_{\mathrm{Q}}$, of galaxies in EAGLE by tracking their $u^{*}-r^{*}$ (intrinsic) colour, specific star formation rates (SSFR) and stellar mass history through redshift. This is achieved using the information stored in the publicly available EAGLE database (McAlpine et al. 2016). We first identify all galaxies that by $z=0$ are quenched, based on their position in either the $u^{*}-r^{*}$ - or SSFR-stellar mass planes. We then compute the difference between the lookback time at which each passive galaxy was last part of the star-forming population, and when they subsequently appeared in the quenched population (based on these two different methodologies, which we describe in more detail below). Note that in either of our definitions we take into account that galaxies in the corresponding planes evolve and thus, the exact definition of star-forming and passive are redshift dependent.

\subsubsection{Color definition of quenching timescale}

We use the $u^{*}-r^{*}-M_{\star}$ plane to create our primary definition (definition 1, referred to as 'D1' hereafter) of quenching timescales, similar to the work of Trayford et al. (2016) and Nelson et al. (2018). To categorise whether a galaxy was part of the blue cloud, red sequence, or green valley, a double-Gaussian distribution was fit to the colour histogram of EAGLE galaxies in bins of stellar mass, at each snapshot. The means and standard deviations in $u^{*}-r^{*}$ colour of the blue cloud and red sequence were estimated and accordingly fitted with a least squares curve fitting function in SciPy. Using these values, we define a galaxy to be quenched if $1.5 \sigma$ above the red-Gaussian mean in a given $M_{\star}$ bin (and snapshot), and star-forming if $1.5 \sigma$ below the blue-Gaussian mean colour in a given $M_{\star}$ bin (and snapshot), according to Eqs. 1 and 2, respectively. Using this definition at $z=0$, we identify 3,054 quenched galaxies, 2,871 galaxies in the process of quenching, and 7,273 star-forming galaxies. This selection process is illustrated in Fig. 1. 


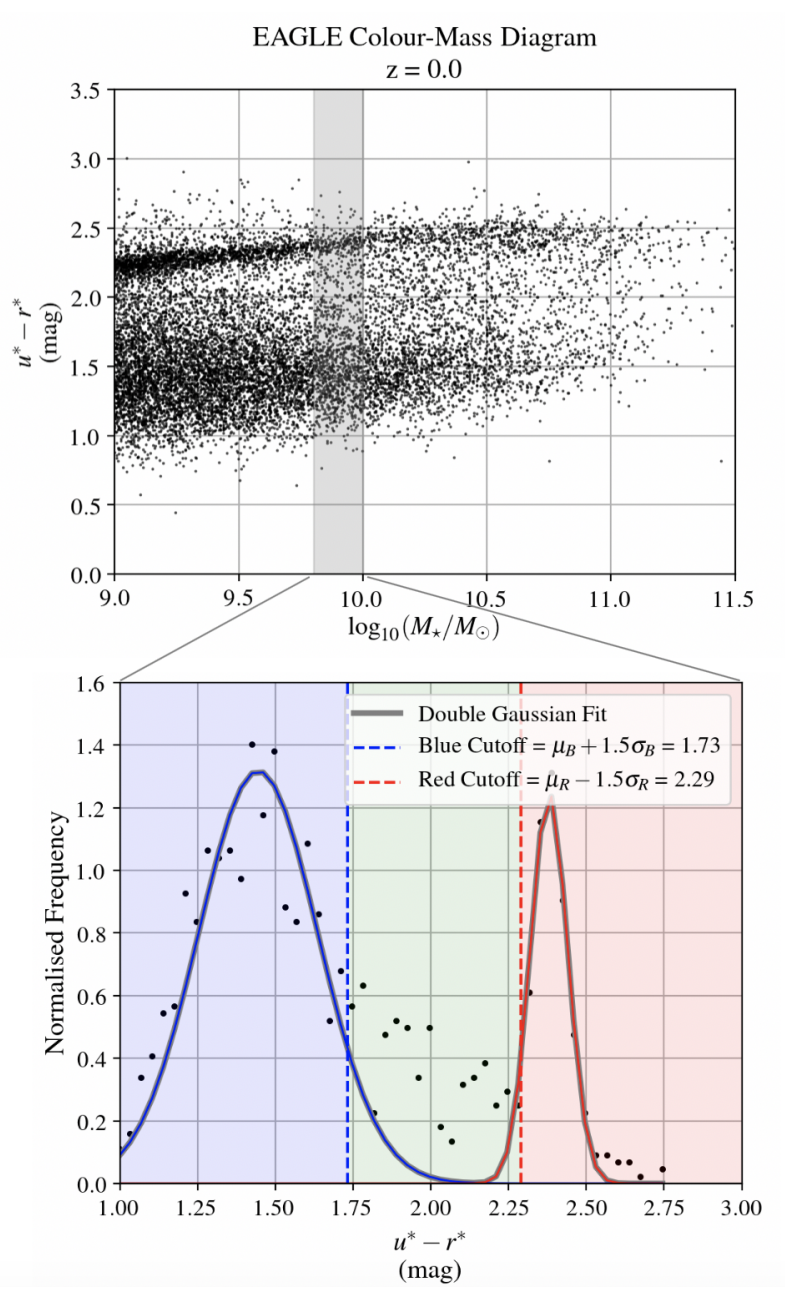

Figure 1. An illustration of the Gaussian fitting criteria to identify the red sequence and blue cloud in binned stellar mass space that we apply to EAGLE. Each population was defined by its mean $\pm 1.5 \sigma$. The region between these two populations defines the green valley (see $\S 2.2 .1$ for details).

$$
\begin{aligned}
& u^{*}-r^{*} \text { blue, cutoff }=\mu_{\mathrm{u}^{*}-\mathrm{r}^{*}}, \text { blue }+1.5 \sigma_{\mathrm{u}^{*}-\mathrm{r}^{*}}, \text { blue } \\
& u^{*}-r^{*} \text { red, cutoff }=\mu_{\mathrm{u}^{*}-\mathrm{r}^{*}}, \text { red } \\
&-1.5 \sigma_{\mathrm{u}^{*}-\mathrm{r}^{*}}, \text { red }
\end{aligned}
$$

\subsubsection{SSFR-stellar mass definition of quenching timescale}

The secondary definition (definition 2, referred to as 'D2' hereafter) for classifying galaxies as star forming or quenched used their SSFRs directly, instead of using galaxy colour as a proxy of star formation activity. The SSFR- $M_{\star}$ plane produces a less well defined population bimodality, which made producing robust definitions somewhat subjective (Wyder et al. 2007; Ciambur et al. 2013; Davies et al. 2016a, 2018a). For these reasons, we consider D1 to be more robust and choose it to be our primary definition (especially at high stellar mass values), since we could distinguish the two populations with confidence. We include D2 for completeness, and remarkably find our conclusions to be largely consistent between the two definitions.

The method we adopt for D2 is based on the po-

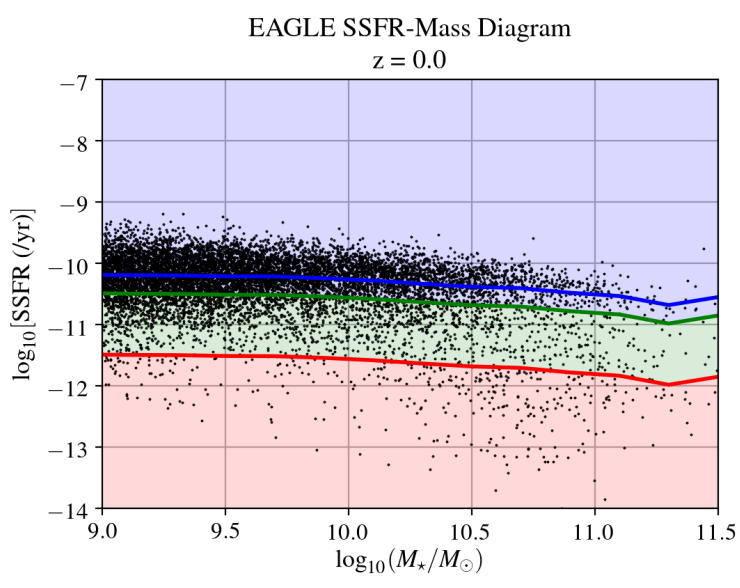

Figure 2. An illustration of the SSFR- $M_{\star}$ criteria for the red sequence and blue cloud in binned stellar mass space. Each population was defined by cutoffs in terms of the position of the main sequence at each stellar mass bin, with values $c_{\text {low }}=0.05$ (red line) and $c_{\text {high }}=0.5$ (green line), below, in between and above these values, galaxies are classified as passive, transitioning and star-forming, respectively (see $§ 2.2 .2$ for details).

sition of the main sequence star formation population, which we quantify with the mean SSFR in bins of stellar mass, $\left\langle\operatorname{SSFR}\left(\mathrm{M}_{\star}\right)\right\rangle$. We then consider 'transitioning' galaxies as those which have $\operatorname{SSFRs}$ between $\mathrm{c}_{\text {low }}\left\langle\operatorname{SSFR}\left(\mathrm{M}_{\star}\right)\right\rangle$ and $c_{\text {high }}\left\langle\operatorname{SSFR}\left(\mathrm{M}_{\star}\right)\right\rangle$. Star-forming and passive galaxies are consequently defined as those with $\operatorname{SSFRs}>c_{\text {high }}\left\langle\operatorname{SSFR}\left(\mathrm{M}_{\star}\right)\right\rangle$ and $\left\langle\mathrm{c}_{\text {low }}\left\langle\operatorname{SSFR}\left(\mathrm{M}_{\star}\right)\right\rangle\right.$, respectively. We adopt values for $\mathrm{c}_{\text {low }}$ and $\mathrm{c}_{\text {high }}$ such that the number of passive, transitioning and star-forming galaxies is similar to those obtained with the color-stellar mass selection of $\S 2.2 .1$, in an attempt to match the subjective thresholds and populations over different definitions. As such, we find the most appropriate multiplicative values to be $c_{\text {low }}=0.05$ and $c_{\text {high }}=0.5$. Using this definition at $z=0$, we obtain 3,369 quenched galaxies, 2,226 quenching galaxies, and 7,613 star-forming galaxies. This selection process is illustrated in Fig. 2. Observational papers have adopted values such as $c_{\text {low }}=0.025-0.5$ (Béthermin et al. 2015; Wang et al. 2018; Davies et al. 2018b), and thus, we consider the values adopted here reasonable compared to those in observations.

\subsection{Reconstructing galaxy formation histories}

To reconstruct the formation histories of galaxies, we use the public database of the EAGLE simulations, described in McAlpine et al. (2016). We limit our study to galaxies with stellar masses $M_{\star}>10^{9} \mathrm{M}_{\odot}$, that are considered as "converged" by Schaye et al. (2015). Note that time span between snapshots in EAGLE ranges from $\approx 0.3-1 \mathrm{Gyr}$. Below, we describe the procedure we follow.

For all galaxies at $z=0$, we define their status as a star-forming, quenching, or passive galaxy based on D1 and D2. Quenched galaxies at $z=0$ are identified and tracked back through redshift. This yields a sample size of $n=3,054$ galaxies for D1 and $n=3,369$ galaxies for D2. These galaxies are followed using the merger tree information stored in the database, described in Qu et al. (2017). If multiple progen- 

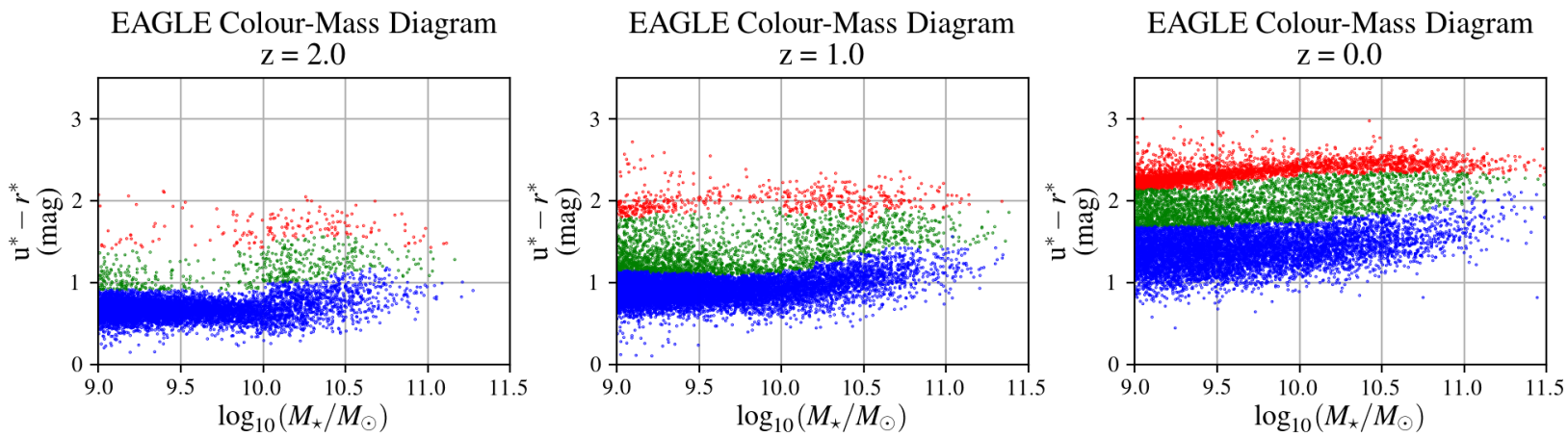

Figure 3. The red sequence developing in EAGLE from $z=2$ to $z=0$, as labelled. We see a scarcity of quenched galaxies at intermediate stellar mass, where galaxies are not massive enough to host a large central SMBH, but are also too massive to be greatly influenced by environmental factors, in concurrence with the work of Davies et al. (2018b) from GAMA observations, and others. Colours indicate star formation status as per $\$ 2.2 .1$ : blue symbols show star-forming galaxies, red symbols show quenched galaxies (part of the red sequence), and green symbols show galaxies in the green valley.
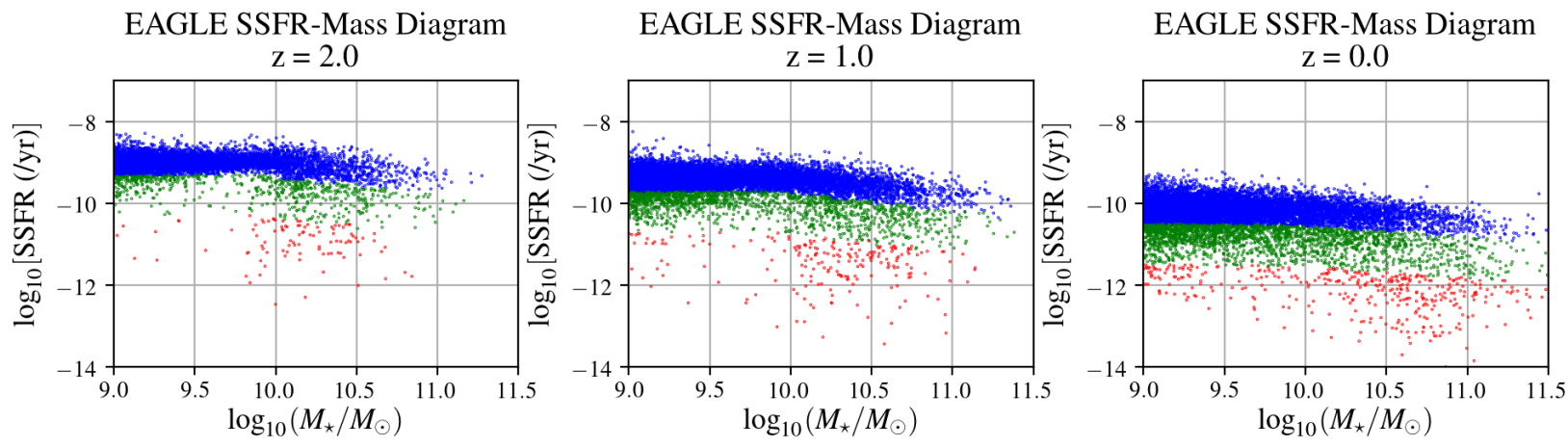

Figure 4. Distribution of galaxies in the SSFR- $M_{\star}$ plane, evolving from $z=2$ to $z=0$, as labelled, in EAGLE. The main sequence of star formation decreases in SSFR over time while more galaxies enter the green transition population moving towards the passive population. The passive population develops from both stellar mass ends with a slight under-density at intermediate stellar mass, similar to Fig. 3, in concurrence with the work of (Davies et al. 2018b) from GAMA observations, and others. Colours indicate star formation status as per $\S 2.2 .2$ : blue symbols show star-forming galaxy (member of main sequence of star formation), red symbols show quenched galaxies (member of the passive cloud), and green symbols show transitioning galaxies.

itor galaxies exist, the most massive (in stellar mass) progenitor in the previous snapshot is selected as the main progenitor, and we continue tracking this branch. Only redshifts below $z=2$ are considered, since at higher redshifts, the passive population is seldom developed, as demonstrated in Fig. 3 for D1 and Fig. 4 for D2. At each snapshot, we record the stellar mass, gas mass (at various apertures), colour, SFR, SMBH mass, group mass and status of central or satellite of each of the $z=0$ quenched galaxies which are being tracked back. All quantities applying to individual galaxies (magnitudes, SFR, stellar mass, gas mass) are taken within a $30 \mathrm{kpc}$ aperture, with the exception of total gas mass (for which we analysed the particle data). To determine whether a galaxy was central or satellite at the time it left the blue cloud, we took the SubGroupNumber of the galaxy at the snapshot it left the blue cloud, and for each snapshot before and after. If the galaxy was considered satellite (SubGroupNumber $>0$ ) at any of these snapshots, we classed it as a satellite. Conversely, if the galaxy was not considered a satellite at any of the 3 mentioned snapshots, we classed it as central. Using 3 snapshots to consider for classification avoids some identification issues with the FOF algorithm in EAGLE - those galaxies falling into a larger halo may not yet be considered a satellite of this system, while they are actually being physically affected by the larger group (see discussion in Trayford et al. 2016).

In addition to the quantities above, we also calculate the group total gas mass, total group gas fraction, $30 \mathrm{kpc}$ group gas fraction, cumulative number of galaxy mergers, and net SMBH accretion rate. The total group gas mass is calculated as the total mass of all gas particles contained in a FOF group. The group gas fraction is then calculated as the ratio between the total gas mass and the dark matter+baryon mass of each group. The $30 \mathrm{kpc}$ group gas fraction is calculated as the sum of all intra- $30 \mathrm{kpc}$ gas for each subhalo in a group, divided by the total group mass. Based on these two definitions we could then define a 3rd gas fraction: the "outer" or "halo" gas fraction, equal to the total group gas fraction subtract the $30 \mathrm{kpc}$ group gas fraction. The cumulative number of mergers is calculated by recording the number of times a galaxy had multiple progenitors in the range $z=2$ to $z=0$, requiring that the secondary-to-primary galaxy stellar mass ratio be no larger than 10; thus, we only consider minor and major merger events. We remind the reader that each progenitor galaxy was required to be above the stellar mass threshold of $M_{\star}=10^{9} M_{\odot}$. The net SMBH 


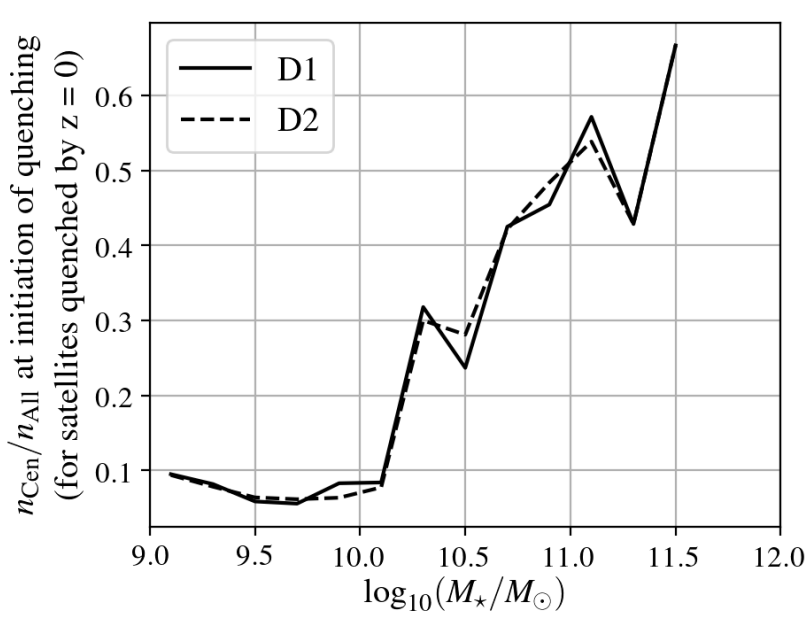

Figure 5. The proportion of quenched satellite galaxies at $z=0$, which were classed as central around the time they left the blue cloud, as a function of stellar mass. The proportion increases significantly with stellar mass for both D1 (solid line) and D2 (dashed line) definitions, indicating that the physics that quenched the high-mass satellite galaxies is likely the same as that of massive central galaxies.

accretion rate was calculated based on the change in $\mathrm{SMBH}$ mass within over 4 snapshots about the time the galaxy left the blue cloud, divided by relevant lookback time difference between the snapshots concerned. We included multiple snapshots to calculate the SMBH accretion rate to avoid stochasticity significantly affecting the derived values. We also tested other definitions of SMBH accretion rates, such as the amount of mass the SMBH grew while the galaxy was transitioning the green valley, divided by that timescale, or the dynamical timescale of the central $3 \mathrm{kpc}$ stellar overdensity. We found our conclusions to be robust under these different definitions (see $\S 3.4$ ). Once each of the mentioned quantities was calculated, we processed the history of each galaxy to determine at which snapshot the galaxy was:

(i) First considered quenched for D1 \& D2, and:

(ii) Last considered star-forming for D1 \& D2.

The difference between the lookback times of these snapshots was taken as the quenching timescale of the given galaxy: the time it took for the galaxy to traverse the transition region, for $\mathrm{D} 1$ and $\mathrm{D} 2$, respectively. Note that because we are using snapshots to define quenching timescales, our measurements are limited to the cadence of the snapshots in EAGLE, which prevents us from measuring quenching timescales much smaller than $1 \mathrm{Gyr}$ (unless quenching occurred at higher redshift).

\section{QUENCHING TIMESCALES OF GALAXIES AND THEIR PHYSICAL ORIGIN}

The mechanisms which cause star formation quenching may act over different timescales, and also leave different imprints on galaxies. Thus, the quenching timescales of galaxies were used here to probe the physical mechanisms of star formation quenching, and ultimately galaxy bimodality. The results to follow include quenching timescale distributions, with populations separated based on their condition of central or satellite galaxy, as well as other properties, which we identify with certain mechanisms of quenching. One should note that the exact values of the quenching timescales are subject to the exact definition of green valley/transitioning region. However, we generally find that the trends presented here are robust to these different definitions. All quantities (e.g. stellar mass, group gas fraction, merger count, unless otherwise stated) are taken at the snapshot the given galaxy left the star-forming population in order to connect to the physical origin of the quenching. In addition, the classification of central or satellite galaxy is taken at the time the galaxy left the star-forming population. This distinction is especially important for passive satellite galaxies at $z=0$, as a significant fraction of them were centrals at the time they left the star-forming sequence (see Fig. 5).

\subsection{Quenching timescales of central and satellite galaxies}

Fig. 6 shows the quenching timescale distribution produced by EAGLE galaxies based on the two definitions D1 and D2 outlined in $\S 2.2 .1$ and $\S 2.2 .2$, respectively, split further into low-mass centrals, high-mass centrals, and satellite galaxies. The low stellar mass to high stellar mass threshold for central galaxies was set at $M_{\star}=10^{9.75} M_{\odot}$, so as to split the stellar mass bimodality for centrals which is illustrated in Fig. 7. We find that the D2 definition gives slightly lower quenching timescales compared to the results obtained by the D1 definition. The latter is expected as even in the case of a sudden cease of star formation, the colour transformation would require longer timescales to complete. Nonetheless, the distribution of quenching timescales in each definition appear mostly consistent, which reassure us that the differences in distributions found are robust. The main difference is seen for low mass centrals, where for $\mathrm{D} 1$ the $\tau_{\mathrm{Q}}$ peak is observed at $3-4 \mathrm{Gyr}$, while for D2 there is a distinct knee (as opposed to a peak) in the distribution, a symptom of the systematically reduced quenching timescales we see for D2. Overall, the median quenching timescales for all selections shown fall in the 1.4Gyr - 3.0Gyr range, which agrees well quantitatively with the measurements reported by Nelson et al. (2018) using the Illustris-TNG simulations.

We interpret the low-mass central population to represent lonesome central galaxies in lower mass halos. For these galaxies, much longer 3-4Gyr timescales are commonplace. Since we do not expect environmental effects or AGN activity to be significant for these galaxies (see $\S 3.3$ and $\S 3.4$ ), it is clear that the main mode of star formation quenching in these galaxies is stellar feedback, which appears to act over significantly longer timescales compared to the typical massive central or satellite galaxy. For higher-mass central and satellite galaxies (existing in higher mass halos), we note that multiple factors are expected to be affecting the quenching of these galaxies, and it is therefore more difficult to interpret these histograms without further decoupling. One can note that satellites do exhibit a knee in their distribution at the $3 \mathrm{Gyr}$ mark when compared to high-mass 

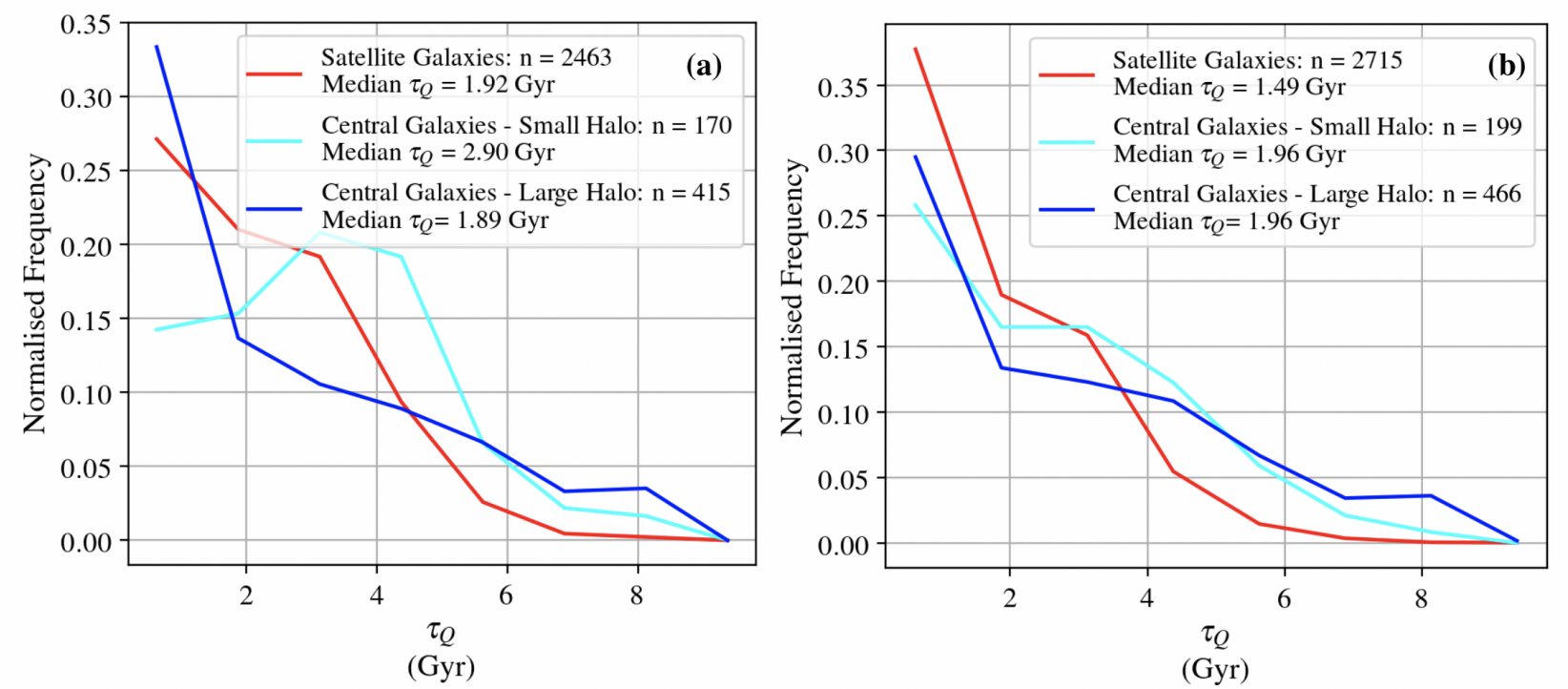

Figure 6. The quenching timescale $\left(\tau_{\mathrm{Q}}\right)$ distribution of $z=0$ passive galaxies, separated into whether they were massive centrals (blue, $M_{\star}>10^{9.75} M_{\odot}$ ), smaller centrals (cyan, $M_{\star}<10^{9.75} M_{\odot}$ ) or satellite galaxies (red) around the time they left the star-forming population, based on D1 (Panel a) and D2 (Panel b) definitions of galaxy state. In general, satellite galaxies show more galaxies to be quenched in the $2-3 \mathrm{Gyr}$ range compared to massive centrals for both $\mathrm{D} 1$ and $\mathrm{D} 2$. The less massive central group exhibits a convincing peak in quenching timescales at $\tau_{\mathrm{Q}}=3.5 \mathrm{Gyr}$ for $\mathrm{D} 1$, and a less prominent but noticeable increase in frequency at $\tau_{\mathrm{Q}}=3.5 \mathrm{Gyr}$ for $\mathrm{D} 2$.
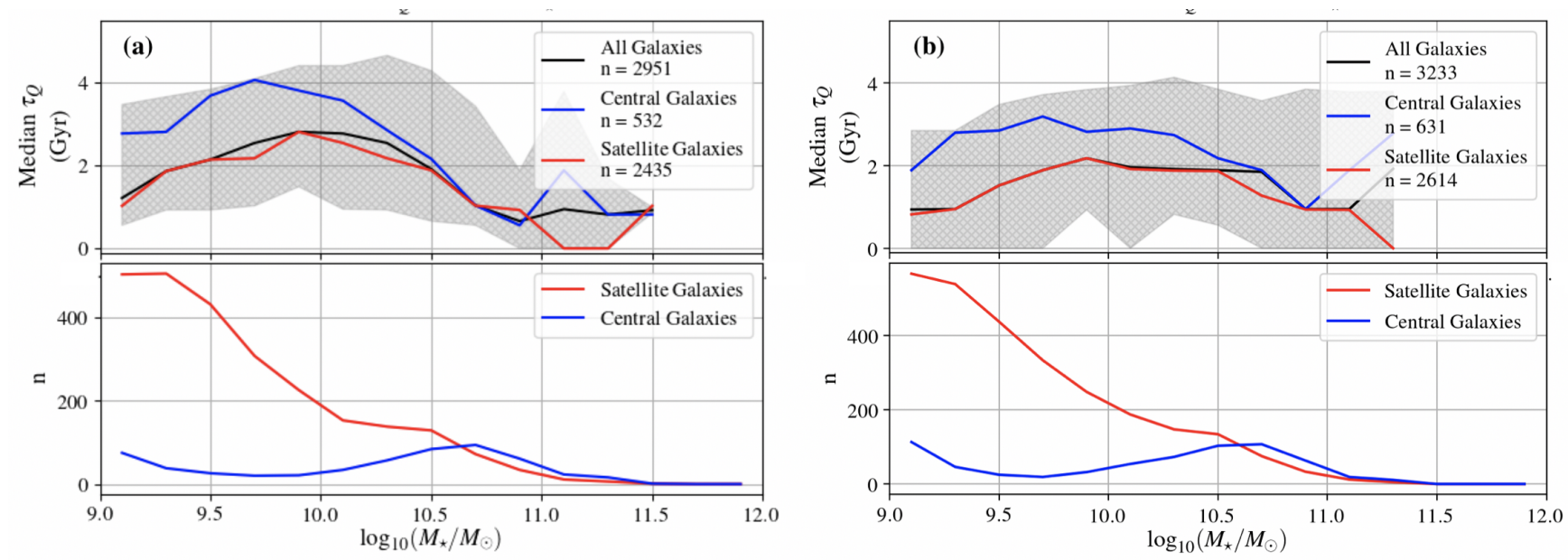

Figure 7. Median quenching timescales of passive galaxies at $z=0$, separating those that were central (blue) and satellite (red) galaxies around the time they left the star-forming population, as a function of stellar mass, for the D1 (Panel a) and D2 (Panel b) definitions of green valley. A peak in quenching timescale is observed at $M_{\star} \approx 10^{9.7} \mathrm{M}_{\odot}$ for all galaxies. The grey shaded region corresponds to the $16^{\text {th }}-84^{\text {th }}$ percentile range for $\tau_{\mathrm{Q}}$ in each stellar mass bin. A positive correlation between stellar mass and quenching timescale is observed at lower stellar masses, while a negative correlation is observed at higher stellar masses. This behavior is seen in both centrals and satellites.

central galaxies, for both D1 and D2 definitions of quenching timescale.

Trayford et al. (2016) studied the pathways in the colour-magnitude diagram of galaxies, and found that lowmass satellites and massive centrals quench first, followed by intermediate mass centrals and satellites. Here, we see that the mechanisms behind those different pathways for centrals and satellites leave a clear imprint in the quenching timescales of these two populations. Hence, in the following subsections, we explore in more detail what drives the distri- bution of quenching timescales, and in which circumstances the quenching timescales of galaxies can elongate or shorten.

\subsection{The dependence of the quenching timescale on stellar mass}

Fig. 7 shows how quenching timescales depend on stellar mass in EAGLE, with these timescales calculated using D1 (Panel (a)) and D2 (Panel (b)) quenching definitions. D1 and D2 give mostly consistent results in terms of distribution shape. We also split galaxies into satellites and centrals, 

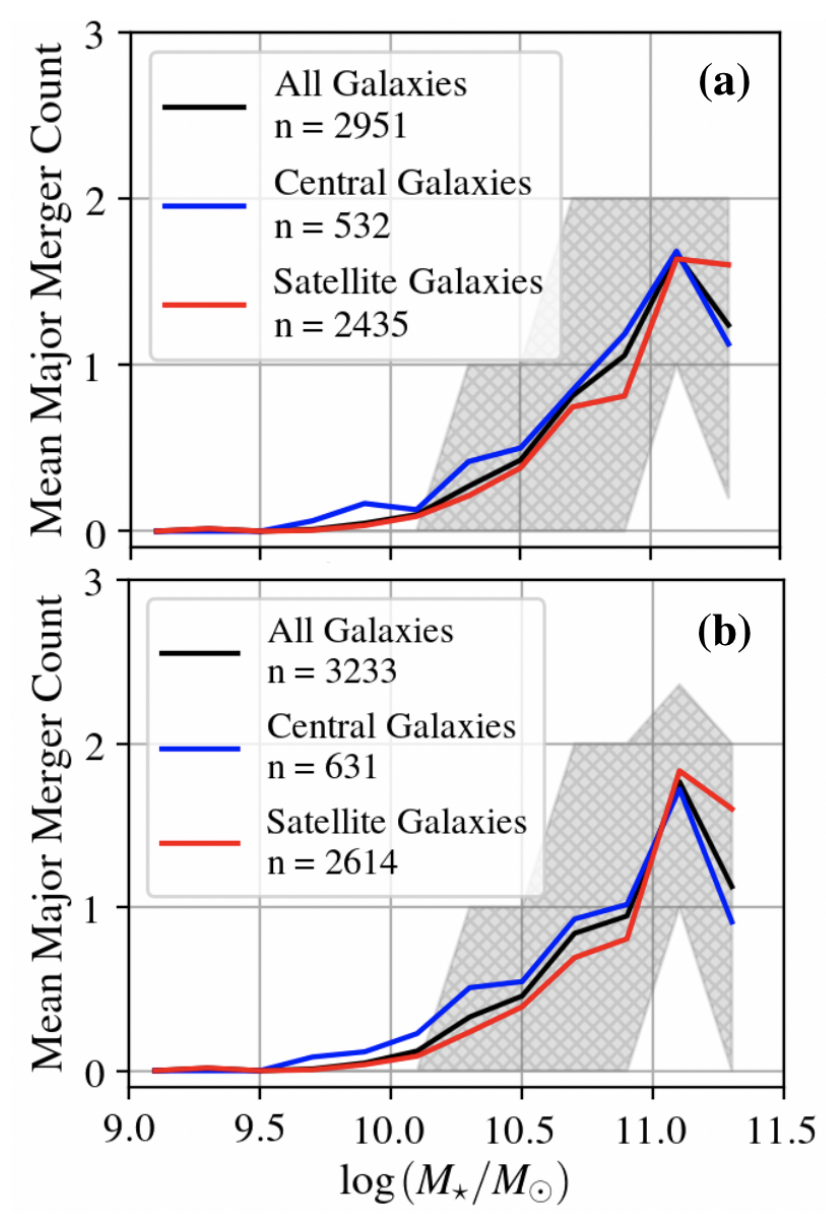

Figure 8. The mean number of major galaxy mergers for passive galaxies at $z=0$ by stellar mass bin, separated into whether they were central or satellite around the time they left the starforming population. We show this for definitions D1 (Panel a) and D2 (Panel b) as a function of stellar mass, only including mergers with mass ratios below 10 . The shaded regions correspond to the $16^{\text {th }}-84^{\text {th }}$ percentile of total merger count in each stellar mass bin (not distinguished between satellite and central galaxies). We seldom observe mergers for low stellar mass galaxies, however at $M_{\star}=10^{10.5} \mathrm{M}_{\odot}$ the mean merger count in each bin picks up and increases rapidly with stellar mass.

as per $\S 2.3$. For both centrals and satellites, we see that there is a peak in quenching timescales at $M_{\star}=10^{9.7} \mathrm{M}_{\odot}$, which corresponds well with the under-density of quenched galaxies at intermediate stellar mass documented in Trayford et al. (2016) and Fig. 3, as well as the observational findings of Davies et al. (2018b). As we will discuss in detail, we find that the dearth of galaxies at that mass scale is directly a consequence of their quenching timescales being on average longer than galaxies at other mass scales, regardless of whether a galaxy is classified central or satellite.

For central galaxies, we see their quenching timescales to be systematically longer than satellites below $10^{10} \mathrm{M}_{\odot}$, meaning the peak at intermediate stellar mass is less defined for centrals. This corresponds to the lower mass central population in Fig. 6. The quenching timescales of satellites and centrals appear to converge above this stellar mass threshold, indicating that the physics occurring to quench high mass galaxies is not a function of central/satellite classification (and thus less likely to be linked to galaxy environment). The turning point at intermediate stellar mass, however, suggests that there are two possibly mechanisms acting for satellites and centrals at the lower stellar mass end, while there appears to be a different, but unique mechanism responsible for quenching high-mass galaxies. The turning point represents the stellar mass at which gas can accrete and stay in a galaxy most efficiently, where gas content in the host halo is most rich. This is further investigated in $\S 3.3$, in our discussion of group gas fractions.

The bottom Panels of Fig. 7 show the histogram of stellar masses of centrals and satellites galaxies. The frequency of satellite galaxies decreases steadily with increasing stellar mass, while centrals display a double peaked distribution. This double-peaked distribution is connected to the efficiency of baryon collapse and star formation being maximal at stellar masses $\approx 10^{10} \mathrm{M}_{\odot}$ in both simulations and observations (Behroozi et al. 2013; Eckert et al. 2017).

To explain the double-peaked distribution of the central galaxy stellar mass, and to probe the mechanisms behind quenching timescales, we explore the connection between the cumulative number of major galaxy mergers in galaxies and the previously mentioned mass transition scale of $\approx 10^{10} \mathrm{M}_{\odot}$ in Fig. 8. We remind the reader that the mass ratio of each merger was required to be less than 10 for consideration. EAGLE shows a dramatic increase in the number of mergers experienced by galaxies at $M_{\star} \gtrsim 10^{10} \mathrm{M}_{\odot}$, which closely corresponds to the turning point in quenching timescales. This also agrees with observation-based findings, where an increase in the significance of mergers as stellar mass increases has been inferred by Robotham et al. (2014). Above this transition mass, the galaxy population in each stellar mass bin moves from satellite- to central-dominated, and we expect from disk- to bulge- dominated. We did not conduct an in-depth investigation on morphology induced changes in quenching timescales. However, we did investigate $z=0$ morphological factors: kinematic bulge-to-total $(\mathrm{B} / \mathrm{T})$ stellar mass ratio and Sèrsic index (as calculated in Lagos et al. 2018), and found there were no convincing trend indicating that galaxy morphological type influenced the time it took a galaxy to transition in EAGLE. Correa et al. (2018) presented a detailed analysis of the relationship between morphological and color transformation of galaxies in EAGLE, and found that the timescale for galaxies to change their color (similar to our $\tau_{\mathrm{Q}}$ in the case of definition D1) were very weakly correlated, in agreement with out findings. They do, however, find a strong correlation for the time at which galaxies join the red sequence and the morphology of galaxies, with early-types typically having joined much earlier than disk galaxies. Our findings, together with those of Correa et al. (2018), support the scenario of quenching timescales being mostly independent of morphology, but with significant differences in the star formation history of galaxies expected for passive disk and elliptical galaxies.

For the rest of this paper, we focus on investigating in depth the physical drivers behind the complex dependence of $\tau_{\mathrm{Q}}-M_{\star}$ we find for central and satellite galaxies, however we will refer back to this section frequently. 


\subsection{External quenching mechanisms and the connection with quenching timescales}

In this section, we explore the effect of environment on the quenching timescales of both central and satellite passive galaxies. First, we consider the role of the galaxy gas content in star formation quenching, both strongly linked in the "bathtub model" (Davé et al. 2012; Lilly et al. 2013; Schawinski et al. 2014) of galaxy gas inflow and outflow. In this model, any quenching mechanism acts by depleting a galaxy's ISM gas reservoir in some way. Before considering which specific gas-removal mechanisms may be at work, we first look at how the gas content of galaxies and their host halos relates to star formation quenching timescales in EAGLE.

Fig. 9 shows the quenching timescale as a function of the gas fraction of a halo using different definitions of gas: taking the total gas mass of the halo ( $M_{\mathrm{Gas}, \text { Total }}$, solid lines), summing the gas of all subhalos in a group "inside" galaxies $\left(\Sigma_{\mathrm{Halo}}\left(M_{\mathrm{Gas}, 30 \mathrm{kpc}}\right)\right.$, using a spherical aperture of $30 \mathrm{kpc}$; dashed lines), and considering only the gas "outside" galaxies (i.e. subtracting the gas "inside" galaxies from the total; $M_{\text {Gas,Total }}-\Sigma_{\text {Halo }}\left(M_{\text {Gas,30kpc }}\right)$, dot-dashed lines $)$, all then normalised by the halo mass, $M_{\text {Halo }}$. One could argue that the demarcation of gas "inside" and "outside" galaxies at 30kpc is arbitrary, however when using $50 \mathrm{kpc}, 70 \mathrm{kpc}$ and $100 \mathrm{kpc}$ apertures as the threshold radii, we find the results to be consistent to those presented below. We show our results for both centrals and satellites that by $z=0$ are classified as passive according to $\S 2.2$. We remind the reader that these gas fractions and stellar masses are calculated at the time these galaxies leave the star-forming population, and stress that these gas fractions apply to full halos (and resident galaxies adopt the fraction calculated for their halo).

For both central and satellite galaxies (and for both definitions of green valley, D1 and D2) we see that the total group gas fraction (solid lines) and outer group gas fraction (dot-dash lines) show minimal correlation with quenching timescale. This is potentially due to the dual effect of gas in halos being capable of stripping galaxies of their gas through ram pressure stripping, while concurrently offering a gas reservoir for accretion to galaxies and eventual star formation.

However, considering just the gas "inside" galaxies (within the $30 \mathrm{kpc}$ radius) (dashed lines in Fig. 9), we find that there is a strong positive correlation between quenching timescales and the $30 \mathrm{kpc}$ group gas fraction over the full range of $\Sigma_{\text {Halo }}\left(M_{\text {Gas, 30kpc }}\right) / M_{\text {Halo }}$ values, from $10^{-3.5}$ to $10^{-1.5}$ for both central and satellite galaxies, using both D1 and D2 $\tau_{\mathrm{Q}}$ definitions. This is an indication that ISM gas is clearly more important for up-regulating star formation (or more specifically, elongating the time that it takes to cease) than the total gas mass within a halo. The correlation for inner gas fraction is steeper for central galaxies with both D1 and D2, where central galaxies which exist in groups with the highest $30 \mathrm{kpc}$ gas fractions have quenching timescales of $>4 \mathrm{Gyr}$ on average - a hugely significant increase compared to the median for all galaxies at $2 \mathrm{Gyr}$. This is possibly since a large proportion of this $30 \mathrm{kpc}$ gas sum for the halo will exist in the central galaxy. The reader should note that we were not able to easily investigate the effects of hot and cool gas with EAGLE, which could decouple the dual effect of stripping galaxies and feeding them with star-forming gas.

Interestingly, if instead we investigate the galaxy gas fraction, $M_{\mathrm{gas}, 30 \mathrm{kpc}} / M_{\star}$, the trend seen for satellite galaxies in Fig. 9 weakens, particularly for the D2 definition. This means that in the case of satellites, the correlation in Fig. 9 is not driven by individual gas-rich satellites having long quenching timescales, but by satellites living in groups that host gas-rich galaxies. On the other hand, we see that the trends seen in Fig. 9 for centrals remain equally strong if we instead consider the galaxy gas fraction. We continue our investigation by probing how the aforementioned group gas fractions vary as a function of stellar mass for galaxies in Fig. 10, with the aim of interpreting the peak of quenching timescales at intermediate masses discussed in $\S 3.2$. Presented is the gas fraction for those galaxies which were quenched at $z=0$ (according to definitions D1 and D2). We note, however, that including the full EAGLE galaxy population at $z=0$ leads to very similar results.

We find that the total halo gas fraction and outer halo gas fraction for satellites to be systematically higher than centrals, indicating that satellites on average exist in more gas-rich halos; while for the $30 \mathrm{kpc}$ halo gas fraction, we find that satellites have preferentially lower gas content. This is consistent with the observations of Brown et al. (2017), using atomic hydrogen spectral stacking of SDSS galaxies. If we consider the total group gas mass fractions and "outer" group gas fraction, ignoring the systematic offset between satellites and centrals, each increases with increasing stellar mass in both D1 and D2 for the full stellar mass range. This is expected from their larger gravitational potential. In the case of the gas "inside" galaxies, we instead find for both satellites and centrals that there is a peak in gas content at intermediate stellar masses, $\approx 10^{10}-10^{10.3} \mathrm{M}_{\odot}$, at a stellar mass slightly larger, by $\approx 0.3-0.5 \mathrm{dex}$, than that of the peak of the quenching timescales at $M_{\star}=10^{9.7} M_{\odot}$. Above $M_{\star} \approx 10^{10.3} \mathrm{M}_{\odot}$, group inner gas fractions start to decrease with increasing stellar mass. Recently, Janowiecki et al. (2017) found in observations that central galaxies of stellar masses $\approx 10^{10.2} \mathrm{M}_{\odot}$ living in groups are more gas-rich than their isolated counterparts of the same stellar mass. Another related observational trend is the one of gas-rich centrals to preferentially live in gas-rich halos (typically referred to as galaxy conformity; Wang et al. 2015). In the context of our results, satellite galaxies in these groups would be the ones that would take the longest to quench, reinforcing the idea that satellites in groups of gas-rich galaxies are the least prone to quenching. Remarkably, the peak of quenching timescales takes place at approximately the same stellar mass as the point in which most of the gas mass in a group goes from being dominated by gas "inside" to gas "outside" galaxies, $M_{\star} \approx 10^{9.7} \mathrm{M}_{\odot}$. AGN activity at stellar masses above the group inner gas fraction peak starts to be efficient enough to keep the gas halos hot and also eject gas from galaxies, as is investigated in $\S 3.4$.

Now, we investigate how the mass of a galaxy in comparison to its host halo can influence star formation quenching. On average, we would expect relatively small galaxies to be more prone to environmental effects from neighbouring large galaxies in the same host halo, discussed at length in Peng et al. (2012) and Davies et al. (2018b). This is because low mass galaxies lack the self-gravity needed to com- 

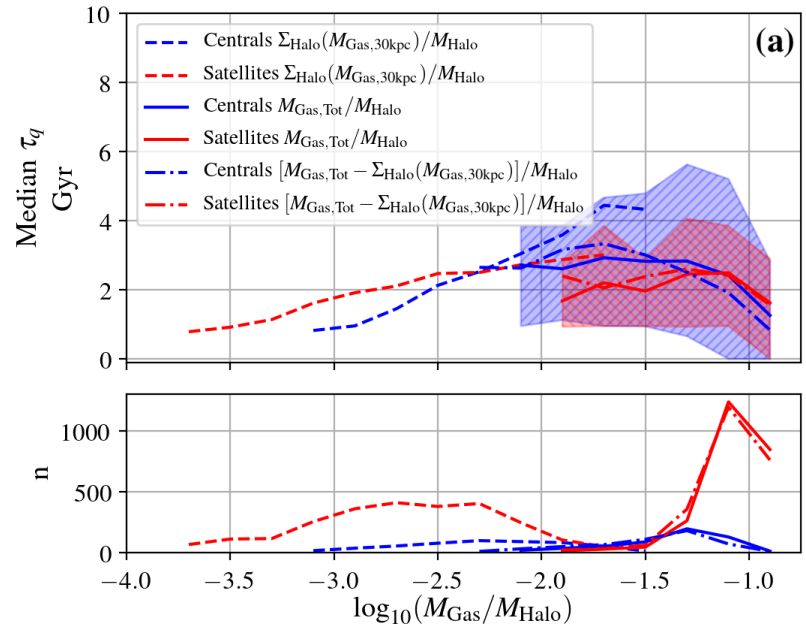
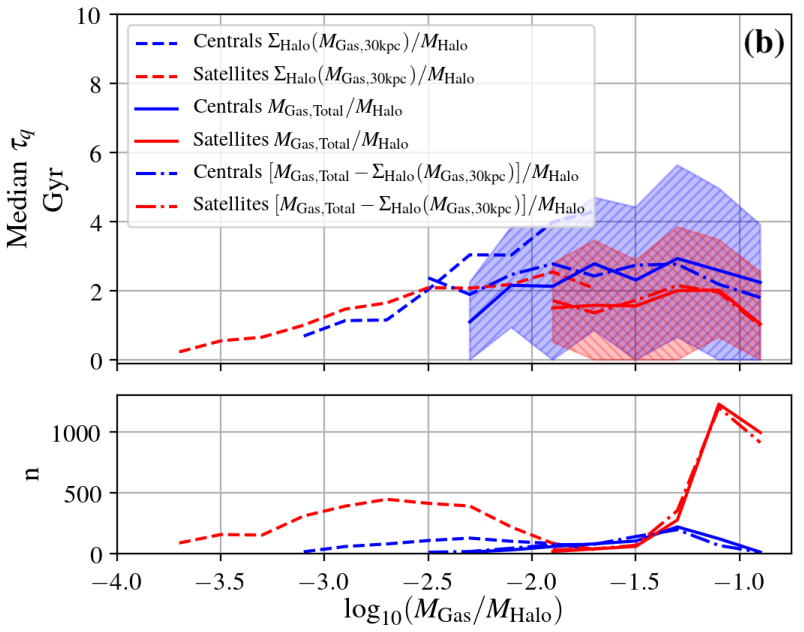

Figure 9. The median quenching timescale of $z=0$ passive galaxies, separated into whether they were centrals (blue) or satellites (red) around the time they left the star-forming population, for the D1 (Panel a) and D2 (Panel b) definitions of green valley, as a function of various group gas fractions. The group gas fractions for each galaxy are calculated by summing the gas mass of each subhalo that belongs to the same group (in the relevant aperture), and normalising with the FOF halo mass. We define "gas mass" using three different criteria: total gas mass (the sum of all gas particles in the FOF halo; solid lines), gas "inside" galaxies (defined as the gas mass within 30 kpc of the center of each subhalo; dashed lines), and finally the gas "outside" galaxies (total gas mass subtracting the gas mass "inside" galaxies; dash-dot lines). Shaded regions correspond to the $16^{\text {th }}-84^{\text {th }}$ percentile range of quenching timescales fraction in each gas fraction bin, for total gas mass fraction only.
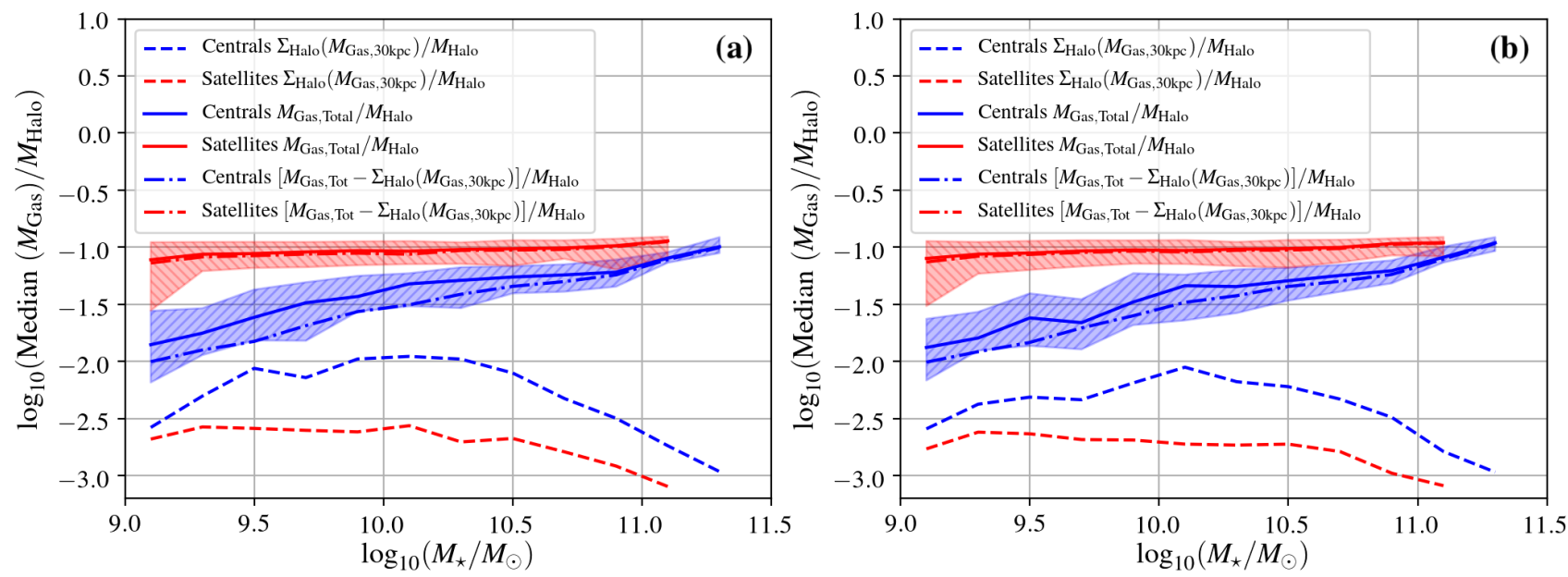

Figure 10. The median group gas fraction of $z=0$ passive galaxies as a function of stellar mass, separating them by whether they were centrals (blue) or satellites (red) around the time they left the star-forming population, for the D1 (Panel a) and D2 (Panel b) definitions of green valley. Here we show the same gas fractions as in Fig. 9. Shaded regions correspond to the $16^{\text {th }}-84^{\text {th }}$ percentile range of total group gas fraction in each stellar mass bin.

pensate for the pressure of the intra-halo gas medium, and are therefore likely to be stripped of their star-forming gas. Empirical estimates of the quenching timescales of satellite galaxies indicate longer timescales as the mass of the satellite increases (Fillingham et al. 2015). To explore this in EAGLE, we use the fraction $M_{\star} / M_{\text {Halo }}$, with $M_{\star}$ and $M_{\text {Halo }}$ being the stellar mass and group mass of the host halo in which the galaxy resided before leaving the star-forming population, to probe whether relatively small satellite galaxies in a halo are quenched over different timescales compared to more massive satellites. In addition, we extend the investi- gation to galaxies that were centrals the time they left the star-forming population.

Fig. 11 shows clearly, for both D1 and D2, that the quenching timescales of satellite galaxies are positively correlated with the $M_{\star} / M_{\text {Halo }}$ ratio for lower stellar-halo mass ratios below $M_{\star} / M_{\text {Halo }}=10^{-3}$. Above this point, quenching timescales decrease. One should note that there are very few satellite galaxies above this mass ratio, and those that do exist are more likely to behave like centrals (as shown in $\S 3.2$ ). For central galaxies, this is harder to ascertain since the $M_{\star} / M_{\text {halo }}$ ratio spans a much narrower range, as expected from the tight stellar-halo mass relation of cen- 

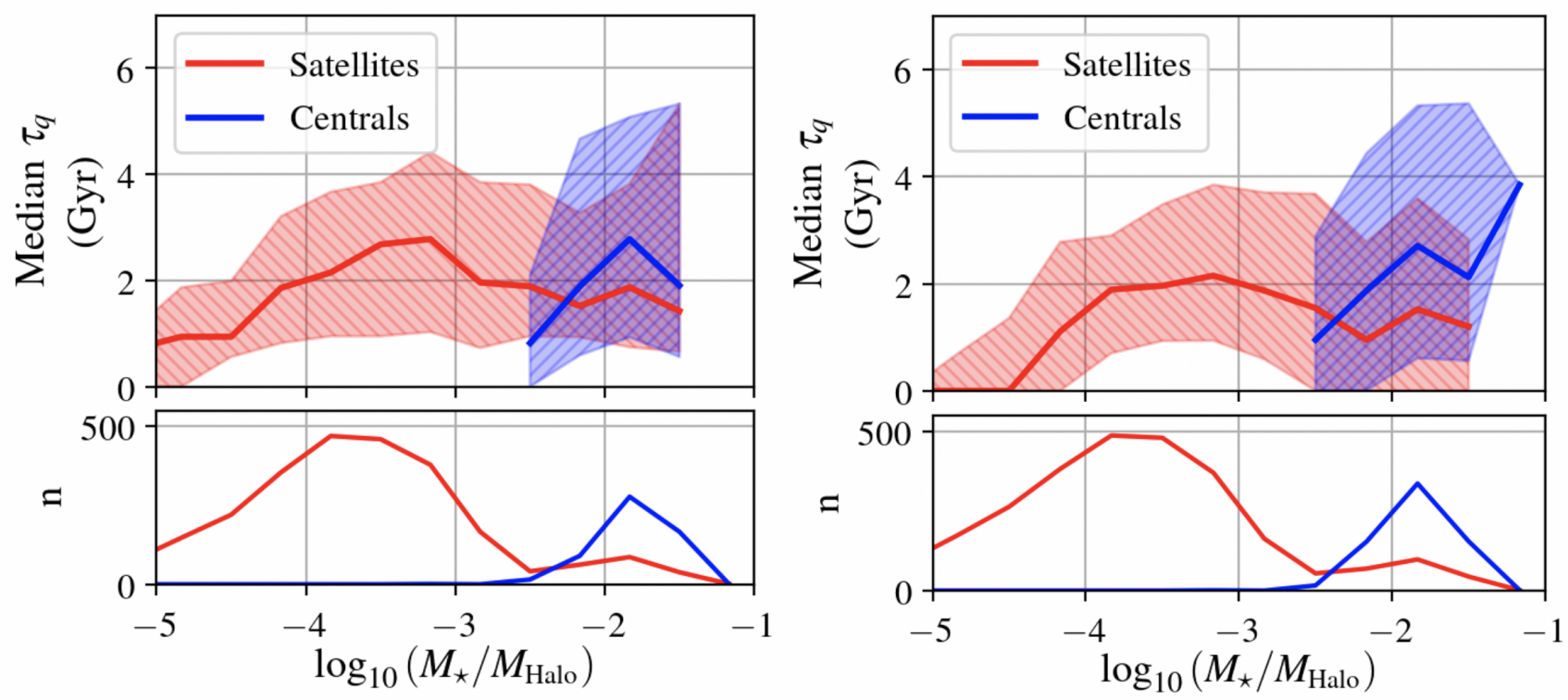

Figure 11. The median quenching timescale of the $z=0$ passive galaxies, separating them into whether they were centrals (blue) or satellites (red) around the time they left the star-forming population, for the D1 (Panel a) and D2 (Panel b) definitions, as a function of the galaxy-halo mass ratio: $M_{\star} / M_{\text {Halo }}$. We define this quantity by using the host halo mass and stellar mass of the galaxies at the redshift when the galaxy left the star-forming population. Shaded regions correspond to the $16^{\text {th }}-84^{\text {th }}$ percentile of $\tau_{\mathrm{Q}}$ in each stellar-halo mass ratio bin. Galaxies more massive relative to their halo typically exhibit longer quenching timescales, this trend being especially obvious for satellite galaxies.

tral galaxies in EAGLE (Schaye et al. 2015; Guo et al. 2016). This said, it appears for both D1 and D2 that quenching timescales increase for central galaxies which are more massive relative to their halo, before decreasing at the highest mass ratio bin for $\mathrm{D} 1$, at $M_{\star} / M_{\text {Halo }} \approx 10^{-2}$.

Short quenching timescales, $\lesssim 1 \mathrm{Gyr}$ dominate the satellite galaxies that are less massive compared to their host halo, while stellar mass-to-halo mass ratios in excess of $10^{-3.5}$ tend to be associated to quenching timescales $\gtrsim 2 \mathrm{Gyr}$. We remind the reader that for this investigation we imposed a stellar mass floor of $10^{9} \mathrm{M}_{\odot}$, and as such the range of $M_{\star} / M_{\text {Halo }}$ is likely limited on the lower end. Based on this evidence, we can conclude that in EAGLE, smaller satellite galaxies in larger halos tend to quench more quickly on average than larger galaxies which are more dominant in their halo. Physically, one could argue that this effect is likely due to the stripping of gas in these smaller galaxies from intrahalo gas pressure, acting to quickly reduce star formation rates and trigger the colour transformation. These conclusions are consistent against D1 and D2 definitions (Panel $\mathrm{a}$ and Panel $\mathrm{b}$ respectively). Note that both the increasing gas fraction with stellar mass and the implied larger stellarto-halo mass ratio with increasing stellar mass for satellites work towards increasing the quenching timescale with increasing stellar mass in this population. Thus, the trend seen in Fig. 7 at $M_{\star} \lesssim 10^{10} \mathrm{M}_{\odot}$ is well explained by the environmental trends reported here. The stellar mass turning point in satellites occurs directly before the "inner" galaxy gas fraction starts to decrease (Fig. 10).

Despite the small dynamic range in $M_{\star} / M_{\text {Halo }}$ obtained for central galaxies in EAGLE, we see that there is a tendency for the quenching timescale to increase with the stellar-tohalo mass ratio, which is more evident for the D2 defini- tion of green valley. This suggests that the scatter of the stellar-halo mass relation is related to how quickly central galaxies quench at fixed halo mass, which in turn correlates with how gas-rich a halo is, and how much SMBH accretion a galaxy has experienced (see discussion in $\S 3.4$ ). Future surveys probing the HI content of galaxies and halos in the local Universe will be able to unveil any systematic effect of the gas fraction of halos on the halo-stellar mass relation and the frequency of green valley galaxies. Those surveys include those to be carried out by the Australian and South African Square Kilometre Array (Blyth et al. 2015).

Recently, Davies et al. (2018b) showed, using the GAMA survey (Driver et al. 2009), that the quenched fraction of satellite galaxies at fixed halo mass increases steeply with the ratio of central-to-satellite stellar mass. They defined quenched galaxies with a method analogous to D2 (using SSFR as the indicator of galaxy transformation). Because the stellar mass of central galaxies is well correlated with the host halo mass, one would expect a similar correlation to exist between the passive fraction and the host haloto-satellite mass ratio. That result naturally fits with our findings with EAGLE and our interpretations, which is that the quenching timescales significantly lengthen for satellites that are massive with respect to their host halo. The latter is expected to have an impact on the overall passive fraction in a way that there are less passive satellites of high stellarto-host halo mass ratios. Thus, these new results of Davies et al. (2018b) (submitted) provide observational evidence for our findings here. 

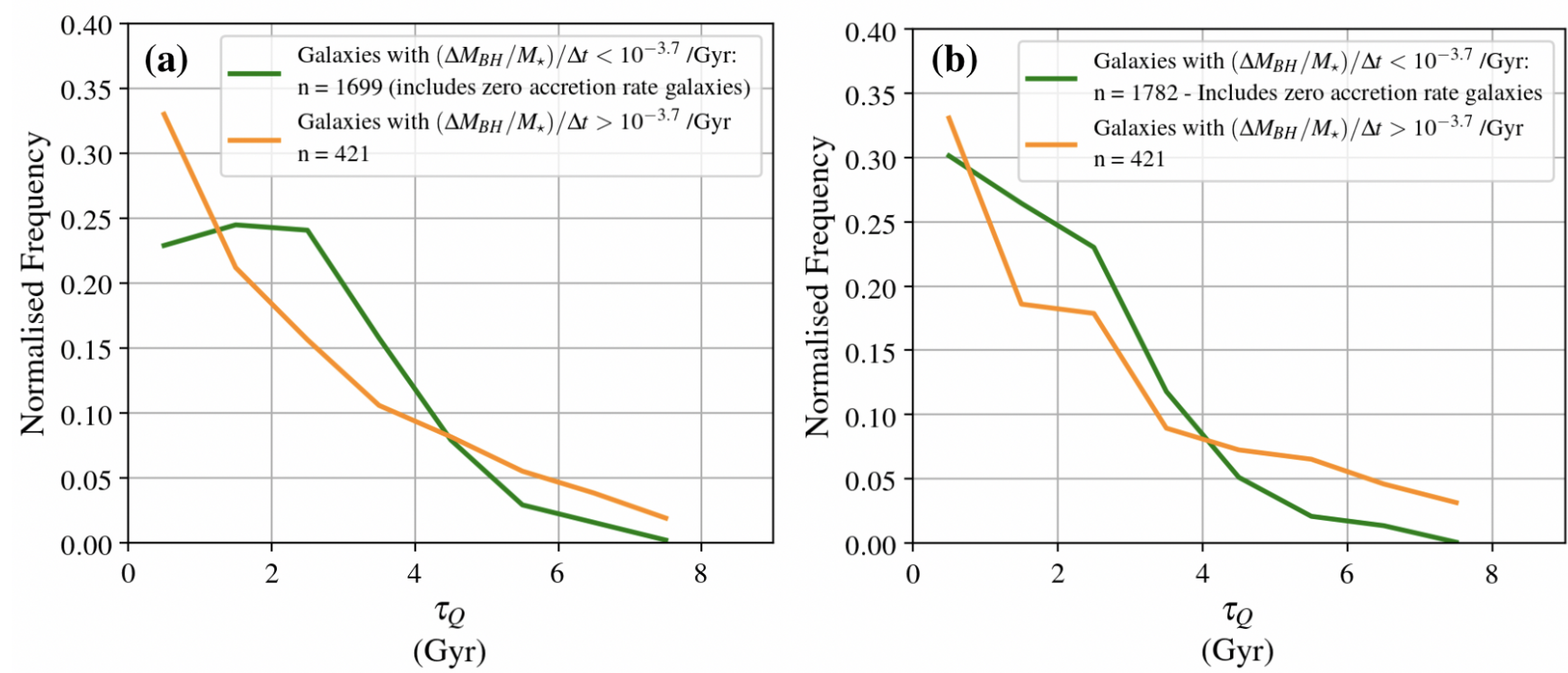

Figure 12. The quenching timescale distribution for all galaxies in bins of high SMBH accretion rate (orange) vs zero- and low-SMBH accretion rate galaxies (green) for both D1 (Panel a) and D2 (Panel b). For zero and low-SMBH accretion rate galaxies the result appear similar, and as such we include these galaxies in the same bin. Roughly half of all galaxies would exhibit a non-zero SMBH accretion rate, and the threshold for low- vs. high-SMBH accretion rate was set at the median accretion rate of the non-zero SMBH accretion rate galaxies. The low accretion rate bin in both $\mathrm{D} 1$ and $\mathrm{D} 2$ exhibits a more spread $\tau_{\mathrm{Q}}$ distribution, with $\mathrm{D} 1$ low accretion rate galaxies actually exhibiting a peak in quenching timescale frequency at $\approx 2-3 \mathrm{Gyr}$. The high accretion rate bin, in contrast, shows a clear peak at the lowest quenching timescale bin and decreases rapidly at longer quenching timescales for both D1 and D2.
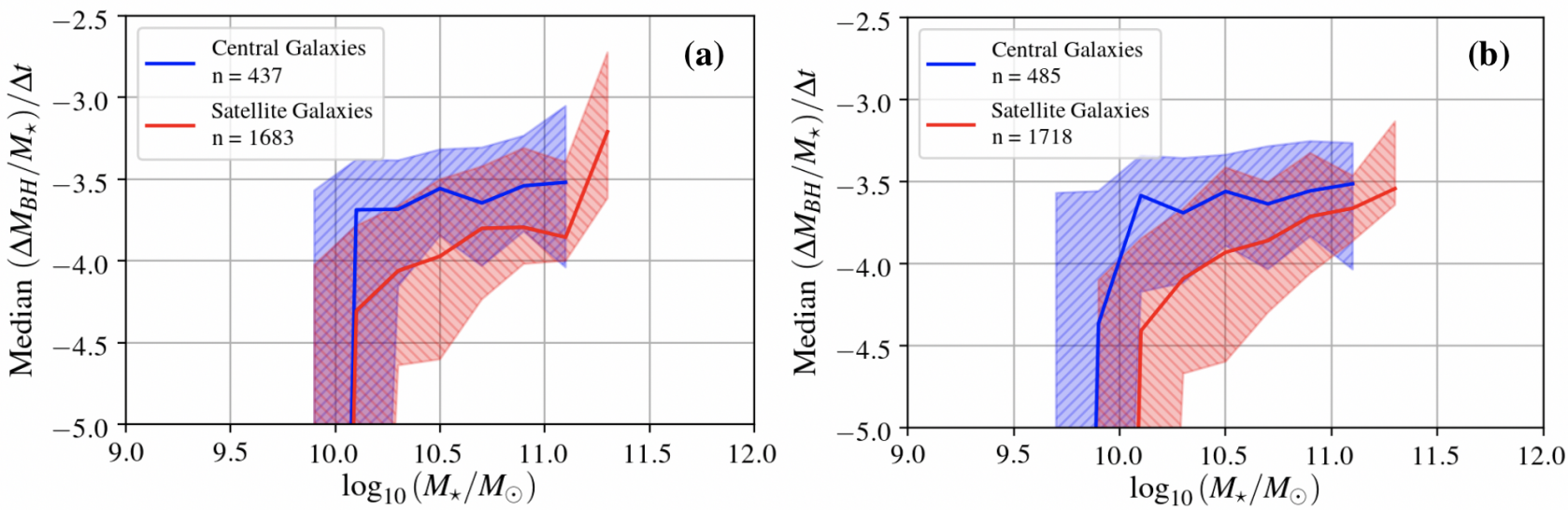

Figure 13. The median SMBH accretion rate in bins of stellar mass for passive galaxies at $z=0$, separated into whether they were satellites (red) or centrals (blue) around the time they left the star-forming population. Panel (a) uses D1, while Panel (b) uses D2. The shaded regions correspond to the $16^{\text {th }}-84^{\text {th }}$ percentile range of SMBH accretion rate in each stellar mass bin. SMBH activity noticeably picks up for both centrals and satellites above $10^{10} M_{\odot}$, the stellar mass regime where we expect satellites and centrals to be quenched by a similar mechanism based on Fig. 7 .

\subsection{AGN activity and quenching timescales}

AGN are known to be capable of quenching star formation due to both heating and ejection of star-forming gas material (see Fabian (2012) for a review on observational evidence of AGN feedback and Bower et al. (2017) for a detailed analysis of AGN feedback in EAGLE). The AGN heating effect in EAGLE depends on how quickly a SMBH can accumulate the necessary energy to heat gas particles to the required temperature (see § 2.1). Thus, AGN feedback in EAGLE critically depends on the SMBH accretion rate.
Fig. 12 shows how the net SMBH accretion rate (normalised by galaxy stellar mass) influences the quenching timescales of central galaxies that at $z=0$ are passive, for both definitions D1 and D2. We split the galaxy population into two bins of net accretion rate: galaxies with high- accretion rate, and galaxies zero- or low- accretion rate. For zero and low- accretion rate galaxies our results appeared similar, and as such we include these galaxies in the same bin. Roughly half of all galaxies would exhibit a non-zero accretion rate. We chose the threshold separating the low- 
vs. high- accretion rate samples to be the median accretion rate of the non-zero accretion rate galaxies. We remind the reader that the net $\mathrm{SMBH}$ accretion rate is calculated as the change in SMBH mass the galaxy experienced over 4 snapshots, around the time the galaxy departed from the star forming population to the passive population. This change in mass was then divided by its stellar mass and by the lookback time difference between the two relevant snapshots.

For both D1 \& D2, we find that the lower SMBH accretion rate galaxies (green lines) exhibit a wider distribution, with a significant tail towards high quenching timescales; while the higher accretion rate galaxies (orange lines) show a much more defined peak at quenching timescales $\lesssim 2 \mathrm{Gyr}$. This indicates that a higher SMBH accretion rate (and corresponding luminosity) acts to shorten quenching timescales through the efficient heating and ejection of inter-stellar gas. This is in line with the findings of Trayford et al. (2016) in EAGLE, which shows that central galaxies with overly massive SMBH are more likely to be passive compared to those with less massive SMBHs. According to our results, the galaxies with massive SMBHs would be more likely to quench faster, and consequently become part of the red sequence.

Recall Fig. 7, where central and satellite galaxies display a peak in quenching timescales at around $10^{9.7} \mathrm{M}_{\odot}$ in EAGLE . To relate back, we now show how the net SMBH accretion rate depends on stellar mass for central and satellite galaxies in Fig. 13. Zero SMBH accretion rate galaxies were included in this context, and accordingly, below $M_{\star}=10^{10} \mathrm{M}_{\odot}$, EAGLE galaxies display a steep drop-off in AGN activity. Our findings agree with the work of Bower et al. (2017), who found that the SMBH accretion rates of galaxies in EAGLE increase rapidly in halos of masses above $10^{12} \mathrm{M}_{\odot}$. This corresponds to the intermediate stellar mass range where we find the rapid increase in AGN activity. This tells us that AGN activity is only likely to affect quenching timescales in galaxies which have higher stellar mass, $M_{\star} \gtrsim 10^{10} \mathrm{M}_{\odot}$. AGN activity picks up rapidly at this stellar mass value, and provides further evidence to the conclusions we make in $\S 3.3$. Thus, we conclude that the turning point in quenching timescale dependence with stellar mass is, at least in part, due to AGN activity which heats the interstellar gas and removes it from the galaxy, at the same time as it keeps the halo gas hot, preventing further cooling. Some galaxies above this mass could be less capable of holding onto their cool interstellar gas reservoir (depending on AGN type and accretion rates).

Interestingly, massive satellite galaxies in EAGLE that are passive by $z=0$ also exhibit an increase in their net $\mathrm{SMBH}$ accretion rate at high stellar masses, which indicates that AGN activity is aiding the quenching of massive satellite galaxies. The latter is necessary to make these galaxies passive, since their environment alone is not enough to drive the cessation of star formation in these galaxies. Also note that many central galaxies that quenched due to the effect of AGN feedback end up being satellites by $z=0$. This fact was illustrated for D1 and D2 in Fig 5.

\subsection{Predictions for the green valley across time}

In coming years, highly complete surveys focused on intermediate-redshift galaxies such as DEVILS (Davies et al. 2018c), could offer insight into the properties of transitioning green-valley galaxies as a function of group properties and redshift. We present here in Fig. 14 the proportion of galaxies in EAGLE that are part of the green valley/ transition region or red sequence/ passive population, based on D1 and D2.

As expected, the overall fraction of passive galaxies increases with time (red lines in Fig. 14), but the fraction of green valley galaxies evolves very weakly with time. This is related to the average timescale for this transitions being rather short. However, some interesting differential behaviour is seen. One of them is that the dependence of the fraction of green valley galaxies with stellar mass for centrals and for definition D1, which at $z=1$ is positively correlated, systematically flattens towards $z=0$, even displaying an inversion of the relation at stellar masses $\gtrsim 10^{10.5} \mathrm{M}_{\odot}$ and at $z \lesssim 0.5$. Interestingly, the fraction of central green valley galaxies in the colour-magnitude diagram at intermediate redshifts $(z \approx 0.5)$ peaks at stellar masses of $\approx 10^{10.2} \mathrm{M}_{\odot}$, due to the quenching timescales being longer at this stellar mass. The flattening is also seen for centrals and the D2 definition, but is less significant than that seen for D1.

For satellites, we also see a rapid increase of the fraction of passive galaxies, which is more striking than that seen for centrals. Interestingly, the green valley fraction of satellites in definition D1 is close to constant at $25 \%$ at $M_{\star} \lesssim 10^{10.2} \mathrm{M}_{\odot}$, with the fraction at higher stellar masses decreasing with time. The inflection stellar mass is due to the mass scale above which we see the quenching timescale quickly decreasing with increasing stellar mass in satellites (see. Fig. 7). Note that this inflection stellar mass also corresponds to when we see a minimum in the fraction of passive satellites, which is clear in both definitions, particularly at intermediate redshifts, $z \approx 0.5-0.75$. For definition $\mathrm{D} 2$, we see again a rather flat fraction of green valley galaxies that varies from $\approx 20 \%$ at $z=1$ to $\approx 10 \%$ at $z=0$. We remind the reader that D1 and D2 definitions of $\tau_{r m Q}$ diverge most noticeably at high stellar masses, where the populations are most difficult to distinguish.

\section{DISCUSSION AND CONCLUSIONS}

In this paper, we have explored the connection between quenching timescales and several galaxy properties in the EAGLE simulations, with the aim of connecting to the different physical processes behind the quenching of star formation in galaxies. We do this using two different definitions (D1, and D2) of quenching timescale. Our study reveals some clear dependencies, which we summarise below.

- Low-mass and high-mass central galaxies exhibit divergent quenching timescale distributions, where low mass centrals were most likely to quench with timescales $\tau_{\mathrm{Q}} \approx 3.5 \mathrm{Gyr}$ in $\mathrm{D} 1$, and there being a noticeable knee in the distribution at this $\tau_{\mathrm{Q}}$ value in $\mathrm{D} 2$. High mass centrals have a sharper peak at $\tau_{\mathrm{Q}} \lesssim 1$.

- Satellite galaxies have a noticeable knee in their $\tau_{\mathrm{Q}}$ distribution at $1.5-3 \mathrm{Gyr}$ compared to high mass central galaxies $(<1.5 \mathrm{Gyr})$ in EAGLE.

- We find satellite and central galaxies in EAGLE (that by $z=0$ are passive) have similar dependencies of their quenching timescales with their stellar mass, except in the 

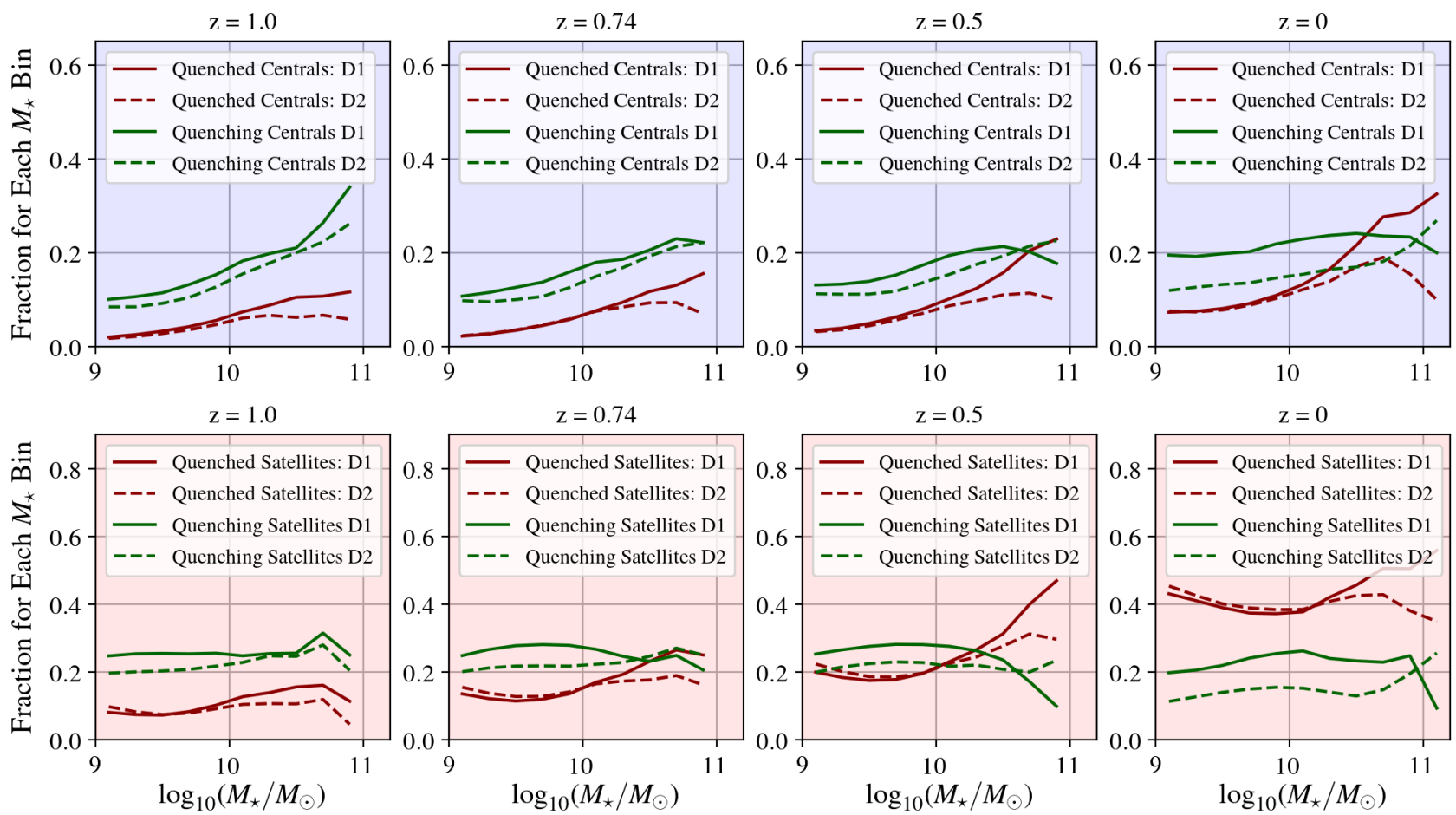

Figure 14. The fraction of galaxies (as a function of stellar mass) that are part of the quenching population or passive population in EAGLE based on D1 and D2, for redshifts between $z=1$ and $z=0$. Over time, for all stellar masses, the quenched fraction of the population increases (corresponding to where galaxies leave the quenching population). This first occurs most notably in the higher stellar mass extremity. At $z=1$ we see a transition population fraction be highest in the higher stellar mass bins, and over redshift, these galaxies transverse to the quenched population, leaving a lower fraction of transition galaxies at higher stellar mass at $z=0$.

lower stellar mass regime. Both populations reveal a quenching timescale that increases with stellar mass up to $M_{\star} \lesssim$ $10^{10} \mathrm{M}_{\odot}$, with the relation reversing at higher stellar masses. This said, at lower stellar mass values, central galaxies have a systematically longer quenching timescale than satellite galaxies, making the $\tau_{\mathrm{Q}}$ peak less dominant for centrals.

- For central galaxies, we find at lower stellar masses $\left(M_{\star}<10^{9.6} M_{\odot}\right)$ that quenching timescales of galaxies are long compared to their satellite counterparts. We attribute the longer quenching timescales to be characteristic of the secular evolution of the stellar population, induced by stellar feedback. At intermediate stellar mass for centrals $\left(10^{9.7} M_{\odot}<M_{\star}<10^{10.3} M_{\odot}\right)$, we find the peak in quenching timescales to be associated with galaxies which are gas-rich in their inner-most $(30 \mathrm{kpc})$ regions, where we see $\tau_{\mathrm{Q}}>3.5 \mathrm{Gyr}$. This result is expected as the gas can readily be converted into stars. In the higher stellar mass regime $M_{\star}>10^{10.3} M_{\odot}$, we observe SMBH accretion rates to increase rapidly along with the cumulative number of major galaxy mergers, two likely interrelated factors. Quenching timescales shorten again in this stellar mass range to $\tau_{\mathrm{Q}}<1.5 \mathrm{Gyr}$, where AGN-induced quenching appears to dominate.

- For satellites in the low stellar mass regime $\left(M_{\star}<\right.$ $\left.10^{9.6} \mathrm{M}_{\odot}\right)$, we find that quenching timescales are particularly short, < 1Gyr, if their stellar mass is low compared to their host halo mass, while stellar-halo mass ratios in excess to $10^{-3.5}$ are linked with lengthened quenching timescales, up to $>2 \mathrm{Gyr}$. We attribute the shortening of satellite quenching timescales relative to centrals in this regime to be induced by ram pressure stripping of satellite galaxies, stifling their star formation. In the intermediate stellar mass range for satellites $\left(10^{9.7} M_{\odot}<M_{\star}<10^{10.3} M_{\odot}\right)$, we find the peak quenching timescales of the group at $\approx 2.5 \mathrm{Gyr}$ which is also associated with the peak group intra-30kpc gas fractions. This points to satellites quenching on long timescales if they are preferentially in groups of gas-rich galaxies. More massive satellites begin to behave very similarly to massive central galaxies, and we find their AGN activity and mean major merger count to be significantly higher for $M_{\star}>10^{10.3} \mathrm{M}_{\odot}$ where quenching timescales shorten again in this stellar mass regime to $\tau_{\mathrm{Q}}<1.5 \mathrm{Gyr}$.

- Regarding the two different definitions of quenching timescale, we find that D1 leads to systematically longer quenching timescales than D2, as expected from the time delay between the ceasing of star formation and the reddening of passively evolving stellar populations. However, all of the conclusions above in terms of centrals/satellites and environment/AGN feedback appeared robust against both of these definitions, which is remarkable.

With the discrete snapshots offered by the EAGLEdatabase, it was impossible to resolve sub-Gyr quenching timescales robustly using the method presented here, and as such, we could not compare the characteristic timescales of environmental and internal quenching mechanisms below that time cadence. However, we can state that AGN and environmental quenching shift the $\tau_{\mathrm{Q}}$ distribution to sub-Gyr scales, and without either of these effects dominating, much longer timescales out to 5-8Gyr are common. These longer 
timescales are mostly associated with galaxies living in gasrich halos, and low mass central galaxies that quench by the action of stellar feedback.

Correa et al. (2018) recently analysed the color vs. morphology transformation of galaxies in EAGLE, finding a poor correlation. This agrees well with our findings, in which a weak or no correlation was found between $\tau_{\mathrm{Q}}$, in either definition, and the kinematic bulge-to-total stellar mass ratio or Sérsic index. However, both our work and that of Correa et al. (2018) show that the colour transformation and quenching timescales can have a complex dependence on galaxy properties, and that centrals and satellites have distinct relationships.

The predictions we present here will be subject to comparison to future observations. Very high spectral resolution spectroscopy, allowing the construction of accurate star formation histories, will be made possible with the James Webb Space Telescope (JWST - Gardner et al. (2006); see Kalirai (2018) for a recent discussion of the science objectives of JWST). Those histories will be key to confirm the quenching timescales reported here for the EAGLE simulations. In addition, highly complete surveys focused on intermediate-redshift galaxies such as DEVILS (Davies et al. 2018c), where most star formation quenching occurs, could also provide valuable insight into the properties of greenvalley galaxies as a function of the group properties. Comparisons between gas-rich and gas-poor groups and the corresponding frequency of green valley galaxies would be useful in assessing the validity of our conclusions, which will be possible with the new generation of SKA (Blyth et al. 2015) in coming years.

\section{Acknowledgements}

We thank Camila Correa for her valuable scientific input and sharing a submitted version of her manuscript. RW is funded by a Postgraduate Research Scholarship from UWA. CL has received funding from a Discovery Early Career Researcher Award (DE150100618) and by the ARC Centre of Excellence for All Sky Astrophysics in 3 Dimensions (ASTRO 3D), through project number CE170100013. We also thanks the Research Collaboration Awards at UWA in its 2018 program, which funded the visit of Dr. James Trayford to ICRAR for two weeks. We acknowledge the Virgo Consortium for making their simulation data available. The EAGLE simulations were performed using the DiRAC-2 facility at Durham, managed by the ICC, and the PRACE facility Curie based in France at TGCC, CEA, Bruyeres-le-Chatel.

\section{REFERENCES}

Baldry I. K., Balogh M. L., Bower R., Glazebrook K., Nichol R. C., 2004, in Allen R. E., Nanopoulos D. V., Pope C. N., eds, American Institute of Physics Conference Series Vol. 743, The New Cosmology: Conference on Strings and Cosmology. pp 106-119 (arXiv:astro-ph/0410603), doi:10.1063/1.1848322

Baldry I. K., Balogh M. L., Bower R. G., Glazebrook K., Nichol R. C., Bamford S. P., Budavari T., 2006, MNRAS, 373, 469 Balogh M. L., Navarro J. F., Morris S. L., 2000, ApJ, 540, 113

Barnes J. E., Hernquist L. E., 1991, ApJ, 370, L65

Behroozi P. S., Wechsler R. H., Conroy C., 2013, ApJ, 770, 57

Béthermin M., et al., 2015, A\&A, 573, A113
Birnboim Y., Dekel A., 2003, MNRAS, 345, 349

Blyth S., et al., 2015, Advancing Astrophysics with the Square Kilometre Array (AASKA14), p. 128

Bower R. G., Benson A. J., Malbon R., Helly J. C., Frenk C. S., Baugh C. M., Cole S., Lacey C. G., 2006, MNRAS, 370, 645

Bower R. G., Schaye J., Frenk C. S., Theuns T., Schaller M., Crain R. A., McAlpine S., 2017, MNRAS, 465, 32

Bremer M. N., et al., 2018, MNRAS, 476, 12

Brown T., et al., 2017, MNRAS, 466, 1275

Ciambur B. C., Kauffmann G., Wuyts S., 2013, MNRAS, 432, 2488

Correa C., Schaye J., Trayford J., 2018, submitted to MNRAS,

Cox T. J., Jonsson P., Primack J. R., Somerville R. S., 2006a, MNRAS, 373, 1013

Cox T. J., Dutta S. N., Di Matteo T., Hernquist L., Hopkins P. F., Robertson B., Springel V., 2006b, ApJ, 650, 791

Crain R. A., et al., 2015, MNRAS, 450, 1937

Croton D. J., et al., 2006, MNRAS, 365, 11

Dalla Vecchia C., Schaye J., 2012, MNRAS, 426, 140

Davé R., Finlator K., Oppenheimer B. D., 2012, MNRAS, 421, 98

Davé R., Thompson R., Hopkins P. F., 2016, MNRAS, 462, 3265

Davies L. J. M., et al., 2016a, MNRAS, 455, 4013

Davies L. J. M., et al., 2016b, MNRAS, 461, 458

Davies L. J. M., et al., 2018a, submitted to MNRAS,

Davies L. J. M., et al., 2018b, submitted to MNRAS,

Davies L. J. M., et al., 2018c, MNRAS, 480, 768

Davis M., Efstathiou G., Frenk C. S., White S. D. M., 1985, ApJ, 292, 371

Di Matteo T., Springel V., Hernquist L., 2005, Nature, 433, 604

Dolag K., Borgani S., Murante G., Springel V., 2009, MNRAS, 399, 497

Driver S. P., et al., 2006, MNRAS, 368, 414

Driver S. P., et al., 2009, Astronomy and Geophysics, 50, 5.12

Dubois Y., Peirani S., Pichon C., Devriendt J., Gavazzi R., Welker C., Volonteri M., 2016, MNRAS, 463, 3948

Eales S. A., et al., 2018, MNRAS,

Eckert K. D., et al., 2017, ApJ, 849, 20

Fabian A. C., 2012, ARA\&A, 50, 455

Fang J. J., Faber S. M., Koo D. C., Dekel A., 2013, ApJ, 776, 63

Fillingham S. P., Cooper M. C., Wheeler C., Garrison-Kimmel S., Boylan-Kolchin M., Bullock J. S., 2015, MNRAS, 454, 2039

Gardner J. P., et al., 2006, in Society of Photo-Optical Instrumentation Engineers (SPIE) Conference Series. p. 62650N, doi: $10.1117 / 12.670492$

Gunn J. E., Gott III J. R., 1972, ApJ, 176, 1

Guo Q., et al., 2016, MNRAS, 461, 3457

Haardt F., Madau P., 2001, in Neumann D. M., Tran J. T. V., eds, Clusters of Galaxies and the High Redshift Universe Observed in X-rays. p. 64 (arXiv:astro-ph/0106018)

Hopkins P. F., 2008, in Kodama T., Yamada T., Aoki K., eds, Vol. 399, Panoramic Views of Galaxy Formation and Evolution. p. 405

Hopkins P. F., Hernquist L., Cox T. J., Di Matteo T., Robertson B., Springel V., 2006, The Astrophysical Journal Supplement Series, 163, 1

Hopkins P. F., Hernquist L., Cox T. J., Kereš D., 2008, The Astrophysical Journal Supplement Series, 175, 356

Janowiecki S., Catinella B., Cortese L., Saintonge A., Brown T., Wang J., 2017, MNRAS, 466, 4795

Jenkins A., 2013, MNRAS, 434, 2094

Jung S. L., Choi H., Wong O. I., Kimm T., Chung A., Yi S. K., 2018, preprint, (arXiv:1809.01684)

Kalirai J., 2018, Contemporary Physics, 59, 251

Kennicutt Jr. R. C., 1998, ApJ, 498, 541

Kereš D., Katz N., Fardal M., Davé R., Weinberg D. H., 2009, MNRAS, 395, 160

Knobel C., et al., 2013, ApJ, 769, 24 
Kocevski D. D., et al., 2012, ApJ, 744, 148

Lagos C. D. P., Cora S. A., Padilla N. D., 2008, MNRAS, 388, 587

Lagos C. d. P., Lacey C. G., Baugh C. M., 2013, MNRAS, 436, 1787

Lagos C. d. P., et al., 2018, MNRAS, 473, 4956

Larson R. B., Tinsley B. M., Caldwell C. N., 1980, ApJ, 237, 692

Lilly S. J., Carollo C. M., Pipino A., Renzini A., Peng Y., 2013, ApJ, 772, 119

Martin D. C., et al., 2007, ApJS, 173, 342

McAlpine S., et al., 2016, Astronomy and Computing, 15, 72

McNamara B. R., et al., 2006, ApJ, 648, 164

Mihos J. C., Hernquist L., 1996, ApJ, 464, 641

Murray N., Quataert E., Thompson T. A., 2005, ApJ, 618, 569

Nelson D., et al., 2018, MNRAS, 475, 624

Noeske K. G., et al., 2007, ApJ, 660, L43

Peng Y.-j., Lilly S. J., Renzini A., Carollo M., 2012, ApJ, 757, 4

Peng Y., Maiolino R., Cochrane R., 2015, Nature, 521, 192

Pillepich A., et al., 2018, MNRAS, 473, 4077

Planck Collaboration et al., 2014, A\&A, 571, A16

Qu Y., et al., 2017, MNRAS, 464, 1659

Robotham A. S. G., et al., 2014, MNRAS, 444, 3986

Salim S., 2014, Serbian Astronomical Journal, 189, 1

Schaller M., Dalla Vecchia C., Schaye J., Bower R. G., Theuns T., Crain R. A., Furlong M., McCarthy I. G., 2015, MNRAS, 454,2277

Schawinski K., et al., 2014, MNRAS, 440, 889

Schaye J., Dalla Vecchia C., 2008, MNRAS, 383, 1210

Schaye J., et al., 2015, MNRAS, 446, 521

Schiminovich D., et al., 2007, ApJS, 173, 315

Sparre M., Springel V., 2017, MNRAS, 470, 3946

Springel V., 2005, MNRAS, 364, 1105

Springel V., Yoshida N., White S. D. M., 2001, New Astron., 6, 79

Springel V., Di Matteo T., Hernquist L., 2005, ApJ, 620, L79

Strateva I., et al., 2001, AJ, 122, 1861

Taylor E. N., et al., 2015, MNRAS, 446, 2144

Toomre A., Toomre J., 1972, ApJ, 178, 623

Trayford J. W., Theuns T., Bower R. G., Crain R. A., Lagos C. d. P., Schaller M., Schaye J., 2016, MNRAS, 460, 3925

Wang J., et al., 2015, MNRAS, 453, 2399

Wang E., et al., 2018, ApJ, 860, 102

Wiersma R. P. C., Schaye J., Theuns T., Dalla Vecchia C., Tornatore L., 2009, MNRAS, 399, 574

Wyder T. K., et al., 2007, ApJS, 173, 293

This paper has been typeset from a $\mathrm{T}_{\mathrm{E}} \mathrm{X} / \mathrm{LAT}_{\mathrm{E}} \mathrm{X}$ file prepared by the author. 